\title{
RESEARCH
}

Open Access

\section{Glioblastoma hijacks microglial gene expression to support tumor growth}

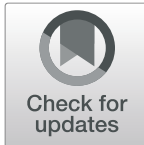

Sybren L. N. Maas ${ }^{1,2 \dagger}$, Erik R. Abels ${ }^{1 \dagger}$, Lieke L. Van De Haar ${ }^{1}$, Xuan Zhang ${ }^{1}$, Liza Morsett ${ }^{4}$, Srinjoy Sil ${ }^{3}$, Joana Guedes ${ }^{4,5}$, Pritha Sen ${ }^{4}$, Shilpa Prabhakar', Suzanne E. Hickman ${ }^{4,6}$, Charles P. Lai ${ }^{1,7}$, David T. Ting ${ }^{3}$, Xandra O. Breakefield ${ }^{1 \dagger}$, Marike L. D. Broekman ${ }^{1,8,9+}$ and Joseph El Khoury ${ }^{4,6^{*}+}$

\begin{abstract}
Background: Glioblastomas are the most common and lethal primary brain tumors. Microglia, the resident immune cells of the brain, survey their environment and respond to pathogens, toxins, and tumors. Glioblastoma cells communicate with microglia, in part by releasing extracellular vesicles (EVs). Despite the presence of large numbers of microglia in glioblastoma, the tumors continue to grow, and these neuroimmune cells appear incapable of keeping the tumor in check. To understand this process, we analyzed gene expression in microglia interacting with glioblastoma cells.

Methods: We used RNASeq of isolated microglia to analyze the expression patterns of genes involved in key microglial functions in mice with glioblastoma. We focused on microglia that had taken up tumor-derived EVs and therefore were within and immediately adjacent to the tumor.

Results: We show that these microglia have downregulated expression of genes involved in sensing tumor cells and tumor-derived danger signals, as well as genes used for tumor killing. In contrast, expression of genes involved in facilitating tumor spread was upregulated. These changes appear to be in part EV-mediated, since intracranial injection of EVs in normal mice led to similar transcriptional changes in microglia. We observed a similar microglial transcriptomic signature when we analyzed datasets from human patients with glioblastoma.

Conclusion: Our data define a microglia Glioblastoma specific phenotype, whereby glioblastomas have hijacked gene expression in the neuroimmune system to favor avoiding tumor sensing, suppressing the immune response, clearing a path for invasion, and enhancing tumor propagation. For further exploration, we developed an interactive online tool at http://www.glioma-microglia.com with all expression data and additional functional and pathway information for each gene.
\end{abstract}

Keywords: Glioblastoma, Glioma, Microglia, Extracellular vesicles, Exosomes, Microvesicles, Macrophages, Sensome, RNASeq, TGF- $\beta$

\footnotetext{
* Correspondence: jelkhoury@mgh.harvard.edu

${ }^{\dagger}$ Sybren L. N. Maas and Erik R. Abels contributed equally to this work. Xandra

O. Breakefield, Marike L. D. Broekman and Joseph El Khoury contributed equally to this work.

${ }^{4}$ Center for Immunology \& Inflammatory Diseases, Massachusetts General Hospital, Harvard Medical School, Boston, MA 02129, USA

${ }^{6}$ Department of Medicine, Massachusetts General Hospital, Harvard Medical School, Boston, MA 02129, USA

Full list of author information is available at the end of the article
}

(C) The Author(s). 2020 Open Access This article is licensed under a Creative Commons Attribution 4.0 International License, which permits use, sharing, adaptation, distribution and reproduction in any medium or format, as long as you give appropriate credit to the original author(s) and the source, provide a link to the Creative Commons licence, and indicate if changes were made. The images or other third party material in this article are included in the article's Creative Commons licence, unless indicated otherwise in a credit line to the material. If material is not included in the article's Creative Commons licence and your intended use is not permitted by statutory regulation or exceeds the permitted use, you will need to obtain permission directly from the copyright holder. To view a copy of this licence, visit http://creativecommons.org/licenses/by/4.0/ The Creative Commons Public Domain Dedication waiver (http://creativecommons.org/publicdomain/zero/1.0/) applies to the data made available in this article, unless otherwise stated in a credit line to the data. 


\section{Background}

Harnessing the power of the immune system to treat cancer has gained significant momentum in recent years. Glioblastomas are diffusely infiltrating tumors of the brain. Because of their invasive nature, total neurosurgical resection of glioblastomas is not possible, resulting in tumor recurrence even following chemo- and radiotherapy [1]. Therefore, new effective treatment strategies for glioblastomas are desperately needed, including therapies utilizing the patients' own immune system [2]. Understanding how glioblastoma cells interact with the immune system is the key to developing immune-based treatments for this tumor [2].

Glioblastomas recruit neighboring resident microglia through the secretion of various chemokines and cytokines [3, 4]. These microglia together with infiltrating monocytes and macrophages can make up to $44 \%$ of the glioblastoma mass $[5,6]$. However, in spite of the presence of large numbers of microglia, monocytes, and macrophages in glioblastoma, the tumors continue to grow, and immune cells appear incapable of controlling such growth. It is accepted that glioblastoma-associated microglia, monocytes, and macrophages play a role in promoting tumor growth [7, 8]. Indeed, depletion of these cells results in reduced glioblastoma invasion and growth in organotypic brain slices and in vivo $[9,10]$. While the evidence that supports this assertion is growing, the exact pathways involved in this tumorsupportive process have not been characterized. Furthermore, the effect(s) of microglia, monocytes, and macrophages that are within the tumor environs versus those in other areas of the tumor-bearing brain but distant from the tumor have not been investigated.

Tumor cells can alter their milieu in part by releasing extracellular vesicles (EVs), including exosomes and microvesicles [11-13]. EVs are a heterogeneous collection of membrane-bound carriers with complex cargoes, including proteins, lipids, and nucleic acids [13-16]. Tumor-derived EV uptake by microglia leads to changes in expression of some genes in these cells as established in vitro $[17,18]$. We have previously visualized such interactions both in vitro and in vivo using a syngeneic mouse glioblastoma model expressing palmitoylated green or red fluorescent proteins (palmGFP and palmtdTomato, respectively) [18-20]. These palmitoylated fluorescent proteins label membranes of tumor cells as well as EVs produced by them (e.g., EV-GFP) [20]. This model allowed us to visualize and isolate microglia, monocytes, and macrophages that had taken up tumorderived EVs in vivo and are therefore closely interacting with glioblastoma cells. In the work presented here, we isolated these microglia, monocytes, and macrophages by fluorescence activated cell sorting (FACS) and analyzed their transcriptomes using bulk RNAseq. To facilitate future analysis of these transcriptomes, we developed an interactive online tool with additional functional and pathway information linked to each gene. To illustrate the usefulness of our dataset and online tool, we performed a focused analysis of microglia. We found that EV-GFP ${ }^{\text {pos }}$ microglia (i.e., present within the tumor) have dysregulated expression of genes in the homeostatic TGF- $\beta$ pathway suggesting a disease specific non-homeostatic phenotype in glioma microglia. Furthermore, genes involved in sensing tumor cells, host defense, and those involved in tumor killing were downregulated, whereas those involved in facilitating tumor spread were upregulated. The evoked role of tumorderived EVs in this microglial transformation was supported by finding similar changes in microglia isolated after uptake of glioma-derived EVs injected intracranially into the brain. Our results were further validated when we analyzed existing bulk and single-cell sequencing datasets of human glioblastoma-associated microglia and found that these microglia displayed similar alterations as observed in the mice. Taken together, these data identify specific changes in the transcriptome of microglia in the presence of glioblastoma that support tumor growth.

\section{Materials and methods \\ Mice}

Animal experimentation was approved by the Massachusetts General Hospital Institution Animal Care and Use Committee. C57BL/6 mice (Charles River Laboratories) were crossed with homozygous C57/ BL6.CCR2 ${ }^{\text {RFP/RFP }}$ knock-in mice [21] to generate heterozygous C57BL6.CCR2 ${ }^{\text {RFP/WT }}$ knock-in mice. Mice were maintained under a 12-h light/dark cycle with access to water and food. Adult mice ranging from 12-18 weeks were used in this study. Male and female mice were randomly assigned to experimental groups. Mice had similar tumor sizes. RNAseq of microglia from male and female animals showed no differences in expression between males and females (data not shown). The 4-week time point was chosen as this is the time point at which mice implanted with GL261 cells first start to develop physical signs and have to be sacrificed per animal welfare guidelines.

\section{Cell culture}

Mouse glioblastoma cell-line GL261 wildtype (NCI Tumor Repository) was cultured in Dulbecco's modified Eagle's medium (DMEM) (Corning) supplemented with $10 \%$ fetal bovine serum (FBS) (Gemini Bioproducts), penicillin (100 units. $\left.\mathrm{ml}^{-1}\right)$, and streptomycin $\left(100 \mu \mathrm{g} \cdot \mathrm{ml}^{-1}\right)$ (Corning). Cells were cultured at $37^{\circ} \mathrm{C}$ in a $5 \% \mathrm{CO} 2$ humidified incubator. Cells were periodically tested for mycoplasma contamination and found negative. 


\section{Stable transduction reporter}

To introduce reporter molecules, the mouse glioblastoma cell-line GL261 wildtype (NCI Tumor Repository) was stably transduced using a CSCW2 lentiviral vector [22] encoding a Gaussia luciferase transmembrane biotin acceptor domain fusion protein (GlucB) and GFP separated by an internal ribosome entry site (IRES) domain [23]. A second transduction was performed using a CSCW2 lentiviral vector encoding palmitoylated GFP for pan membrane-associated GFP expression, including in membrane particles released by these tumor cells [20, 24]. Selection and validation of viral transduction and reporter expression, resulting in the generation of GL261.GlucB-IRES-GFP.palmGFP (GL261.BpalmGFP) cells, were done based on GFP expression using FACS (BD FACSAria II SORP Cell Sorter).

\section{Intracranial tumor implantation}

After anesthetizing the animals using $70 \mu \mathrm{l}$ of a mixture of ketamine (Bioniche Pharma) $\left(17.5 \mathrm{mg} \cdot \mathrm{ml}^{-1}\right.$ ) and xylazine (Santa Cruz Biotechnology) $\left(2.5 \mathrm{mg} \cdot \mathrm{ml}^{-1}\right)$, C57BL6.CCR2 ${ }^{\text {RFP/wt }}$ adult mice (12-18 weeks old) were implanted in the striatum with $1 \times 10^{5}$ GL261.BpalmGFP or GL261 wildtype cells in $2 \mu \mathrm{l}$ plain DMEM using a stereotactic frame. Cells were implanted using the coordinates from lambda $-2 \mathrm{~mm}$ anterior, 0.5 $\mathrm{mm}$ left, and a depth of $2.5 \mathrm{~mm}$ from the skull. Four weeks after implantation, the mice were deeply anesthetized with $120 \mu \mathrm{l}$ of a mixture of ketamine $\left(17.5 \mathrm{mg} \cdot \mathrm{ml}^{-1}\right)$ and xylazine $\left(2.5 \mathrm{mg} \cdot \mathrm{ml}^{-1}\right)$ followed by transcardial perfusion with $50 \mathrm{ml}$ PBS for FACS or 4\% PFA (VWR) for immunohistochemistry using a perfusion pump (Minipump Variable Flow, Fisher Scientific).

\section{EV isolation and intracranial injection}

EVs were isolated from supernatant of GL261.BpalmGFP cultured for $48 \mathrm{~h}$ in DMEM with penicillin (100 units. $\mathrm{ml}^{-1}$ ) and streptomycin $\left(100 \mu \mathrm{g} \cdot \mathrm{ml}^{-1}\right)$ (Corning) and EV-depleted FBS. FBS was depleted of EVs by overnight $(16 \mathrm{~h}$ ) ultracentrifugation at $200.000 \times g$ ( $k$-factor 110.5). EV isolation was done using differential ultracentrifugation protocol consistent of centrifugation of supernatant at $300 \times g$ for $10 \mathrm{~min}, 2000 \times g 10 \mathrm{~min}$, filtering through $0.8 \mu \mathrm{m}$ filter (Sigma), and $100.000 \times g$ ( $k$-factor of 220.1) $120 \mathrm{~min}$ in Quick-Seal $^{\circ}$ Polypropylene Tubes (Beckman) using Type $70 \mathrm{Ti}$ in Optima ${ }^{\mathrm{TM}} \mathrm{XE}$ ultracentrifuge (Beckman) to pellet EVs. EV pellets were concentrated by centrifugation at $100.000 \times g$ ( $k$-factor of 190.7) for 120 min in Thinwall Polypropylene Tubes (Beckman) using MLS-50 Swinging-Bucket Rotor (Beckman) in an Optima ${ }^{\text {TM }}$ MAX-XP Ultracentrifuge (Beckman). Pelleted EVs were resuspended in PBS, and subsequent characterization of EV pellet was performed by size distribution analysis using nanoparticle tracking analysis
(Malvern) and western blot analysis. For western bolt analysis, EV pellets and cells were resuspended in RIPA buffer. Equal amount of protein as measured by Pierce BCA protein assay (Thermo Fisher) was loaded and ran on $10 \%$ SDS-PAGE gel (Thermo Fisher). Proteins were transferred onto nitrocellulose membrane and probed for ALIX (Santa Cruz, sc-53538, 1:200), TSG101 (Abcam, ab125011, 1:500), Flotillin-1 (Abcam, ab133497, 1:500), GAPDH (Millipore, CB1001, 1:1000), and GFP (Thermo Fisher, A-11120, 1:1000).

EV or carrier fluid (PBS) was injected intracranial following identical procedures as described in intracranial tumor implantation method section. Using NTA 2.2 with shutter set at 1000 and gain at 400 , a 1 to 500 dilution of EV concentrate was measured with $>1000$ completed tracks [25]. A total of $3 \mu \mathrm{l}$ with a concentration of $1.4 \mathrm{e} 12$ particles. $\mathrm{ml}^{-1}$ was injected. Microglia were isolated $16 \mathrm{~h}$ after injection of EV or DPBS following procedures as described in methods sections harvesting of brains and preparation of single-cell suspensions and cell staining and FACS.

\section{Immunohistochemistry}

Brains were collected and placed in 4\% PFA for $24 \mathrm{~h}$ and subsequently placed in $25 \%$ sucrose for $48 \mathrm{~h}$. The brains were then frozen in optimal cutting temperature compound (OCT) media (Sakura) in a dry ice bath containing 2-methyl-butanol. Twelve micro cryosections were prepared, placed on glass slides, and stored at $-80^{\circ} \mathrm{C}$. For processing, sections were washed for $10 \mathrm{~min}$ in PBS and permeabilized with $0.5 \%$ Triton-X PBS for $1 \mathrm{~h}$ at room temperature. Sections were blocked for $1 \mathrm{~h}$ at room temperature using 5\% Normal Goat Serum (NGS) (Abcam) in PBS. Subsequently, the sections were labeled with a primary goat antibody and blocked using 5\% Bovine Serum Albumin (BSA; Sigma-Aldrich) in PBS. Primary antibodies were diluted in $1.5 \%$ NGS or $1.5 \%$ BSA. Slides were then incubated with primary antibody solution overnight at $4{ }^{\circ} \mathrm{C}$. After incubation, slides were washed $3 \times 10 \mathrm{~min}$ in PBS. The secondary antibodies were diluted in $1.5 \%$ NGS or $1.5 \%$ BSA. Sections were then incubated with secondary antibody solution for $1 \mathrm{~h}$ at room temperature and subsequently washed $3 \times 10$ min using PBS. DAPI (0.1 $\mu \mathrm{g} \cdot \mathrm{ml}^{-1}$, Thermo Fisher) staining was performed for $30 \mathrm{~min}$ at room temperature. Next, the slides were washed for $10 \mathrm{~min}$ using PBS. Sections were mounted using ProLong Diamond Antifade Mountant (Thermo Fisher). Primary antibodies used were goat-anti-mouse ARG1 (Santa Cruz Biotechnology, sc18354, 1:200), goat-anti-mouse CD74 (Santa Cruz Biotechnology, sc5438, 1:200), rabbit-anti-mouse IBA1 (Wako, 019-19741, 1:1000), and mouse-anti-GFP tag antibody (Thermo Fisher, A-11120, 1:200). Secondary antibodies were donkey-anti-goat IgG Alexa Fluor 647 
(Thermo Fisher, A21447, 1:500), donkey-anti-rabbit IgG Alexa Fluor 405 (Thermo Fisher, A31556, 1:500), and goat-anti-mouse IgG Alexa Fluor 488 (Thermo Fisher, A31560, 1:500).

\section{Microscopy}

Fluorescence microscopy images were acquired on the Zeiss Axio Imager M2 (Carl Zeiss). Confocal images were obtained using the Zeiss LSM 710 inverted confocal microscope.

\section{Harvesting of brains and preparation of single-cell suspensions}

After anesthetizing and perfusing with PBS, brains were removed and processed into single-cell suspension as described [26]. Briefly, brains were cut into small pieces and placed into a GentleMacs ${ }^{\mathrm{Tn}} \mathrm{C}$-tube (Miltenyi Biotech, San Diego, CA, USA) with Roswell Park Memorial Institute (RPMI) 1640 with L-glutamine (no phenol red) medium (Fisher Scientific) containing dispase $\left(2 \mathrm{U} \cdot \mathrm{ml}^{-1}\right)$ (Corning) and collagenase type 3 at a final concentration of $200 \mathrm{U} \cdot \mathrm{ml}^{-1}$ (Worthington Biochemicals). The resulting mixtures were processed using the gentleMACS Dissociator (Miltenyi Biotech) on the brain program settings according to manufacturer's directions. Thus, the brains were subjected to three rounds of dissociation each followed by a period of incubation at $37^{\circ} \mathrm{C}$ for 10 min. DNase I grade II (Roche Applied Science) was added to a final concentration of $40 \mathrm{U} \cdot \mathrm{ml}^{-1}$ and incubated for an additional $10 \mathrm{~min}$ before the final round of dissociation. After dissociation steps, PBS/EDTA containing 5\% FBS was added to inactivate the enzyme mixture, and brain pieces were gently triturated gently, passed through a $100-\mu \mathrm{m}$ filter (Fisher Scientific) and centrifuged at $400 \times g$ for $10 \mathrm{~min}$. Cell pellets were resuspended in $10.5 \mathrm{ml} \mathrm{RPMI} / \mathrm{L}$-glutamine, mixed gently with $4.5 \mathrm{ml}$ physiologic Percoll (Sigma Aldrich), and centrifuged at $850 \times g$ without brake for $40 \mathrm{~min}$. The subsequent pellets were then rinsed in PBS and centrifuged again at $400 \times g$ for $10 \mathrm{~min}$. Red blood cells in the pellets were lysed using RBC lysis buffer (Boston BioProducts) for $2 \mathrm{~min}$ at room temperature followed by a washing step using RPMI/L-glutamine medium. The final cell suspensions were then resuspended in PBS with $0.2 \%$ FBS or in DPBS, $1 \times$ without calcium $\left(\mathrm{Ca}^{2+}\right)$ and magnesium $\left(\mathrm{Mg}^{2+}\right)$ (Corning) supplemented with $2 \mathrm{mM}$ EDTA (Thermo Fisher), and 0.5\% BSA (Sigma Aldrich), followed by staining and FACS. The interval between perfusion to FACS was approximately $5 \mathrm{~h}$.

\section{Cell staining and FACS}

To block non-specific binding of immunoglobulin to the Fc receptors, cells in suspension were incubated for 10 min on ice with TruStain $\mathrm{fcX}^{\mathrm{mm}}$ (anti-mouse CD16/32,
BioLegend, \#101319, clone 93, 1:100). Cell identification was based on levels of expression of CD45 and CD11b (microglia), CD45, CD11b, F4/80, Ly6C, and CCR2 (monocytes/macrophages). For microglia, we used antiCD45-pacificBlue (BioLegend, \#103125, clone 30-F11, 1: 100) and anti-CD11b-Alexa647 (BioLegend, \#101220, clone M1/70, 1:100) for tumor bearing mice. For monocytes/macrophages, anti-CD45-pacificBlue (BioLegend, \#103125, clone 30-F11, 1:100), anti-CD11b-PE-Cy7 (BioLegend, \#101215, clone M1/70, 1:100), anti-Ly6C-BV605 (BioLegend, \#128035, clone HK1.4, 1:500), and anti-F4/ 80-APC (BioLegend, \#123115, clone BM8, 1:75) were used. Cells were stained for $30 \mathrm{~min}$ on ice with gentle mixing every $10 \mathrm{~min}$ by pipetting the mixture up and down. To remove unbound antibodies, cells were centrifuged at $400 \times g$ for $8 \mathrm{~min}$, resuspended in $0.2 \% \mathrm{FBS}$ in PBS, and passed through a $35-\mu \mathrm{m}$ nylon mesh strainer (BD Falcon). Cells were than sorted using a BD FACSAria II SORP Cell Sorter.

\section{RNA isolation and preparation for RNA sequencing}

Cells isolated from brains in all experiments were directly sorted into $1.5 \mathrm{ml}$ Eppendorf (Hauppauge) tubes containing $350 \mu \mathrm{l}$ RLT Plus lysis buffer (Qiagen) at $4{ }^{\circ} \mathrm{C}$. After FACS was completed, the tubes were weighed, and additional RLT Plus was added to the $1.5 \mathrm{ml}$ Eppendorf if the sorted volume was larger than $50 \mu \mathrm{l}$ at a ratio of a maximum of $50 \mu \mathrm{l} 0.2 \%$ FBS PBS to $350 \mu$ RLT Plus buffer. 2-Mercaptoethanol (Sigma) was added to the tubes at a ratio of $10 \mu \mathrm{l}$ per $1 \mathrm{ml}$ of RLT buffer, and RNA was then isolated using the RNeasy Plus Micro kit (Qiagen) and using the total RNA isolation protocol. Eluted RNA was aliquoted and stored at $-80^{\circ} \mathrm{C}$. Before preparation of cDNA fragments for RNA sequencing, RNA concentrations and quality were determined using the Agilent 2100 Bioanalyzer (Agilent Technologies) Pico-chips. cDNA for RNA sequencing was synthesized from RNA aliquots using the SMARTer Ultra Low Input RNA Kit for Sequencing-v3 (Clontech Takara) according to the manufacturer's protocol. A total of $500 \mathrm{pg}$ RNA were used for subsequent library generation. One microliter of a 1:50,000 dilution of ERCC RNA Spike-In Mix (Life Technologies) was added to each sample. Then, firststrand synthesis and tailing of RNA molecules were performed using 3'-SMART CDS primer II A (selecting for poly-A-tails) followed by extension and template switching by reverse transcriptase. Amplified cDNA was purified with $1 \mathrm{x}$ Agencourt AMPure XP beads (Beckman Coulter), in accordance with the SMARTer protocol. The eluted cDNA was stored at $-20^{\circ} \mathrm{C}$. The Nextera ${ }^{\circ}$ XT DNA Library Preparation kit (Illumina) was used for sample barcoding and fragmentation according to the manufacturer's protocol. cDNA samples were thawed, and a total of $1 \mathrm{ng}$ of amplified cDNA was used for the 
enzymatic tagmentation followed by 12 cycles of amplification and unique dual-index barcoding of individual libraries. PCR product was purified with $1.8 \mathrm{x}$ Agencourt AMPure XP beads as detailed in the Nextera XT protocol, omitting the bead-based library normalization step. Library validation and quantification was performed by quantitative PCR using the $\mathrm{SYBR}^{\circ}$ FAST Universal qPCR Kit (KAPA Biosystems). The individual libraries were pooled with equal concentrations, and the pool concentration was re-determined using the KAPA SYBR ${ }^{\circ}$ FAST Universal qPCR Kit. The pool of libraries was subsequently diluted, denatured, and loaded onto the NextSeq 500 sequencer (Illumina) according to the manufacturer's guidelines with the addition of 1\% PhiX Sequencing Control V3 (Illumina). A NextSeq 500/550 High Output v2 kit ( 150 cycles) was used to run 75 -bp pairedend sequencing.

\section{Immunofluorescent quantification}

Zen Pro 2012 (Carl Zeiss) and ImageJ 1.49v (NIH) software packages were used to process the images. For immunofluorescence quantification, the fluorescence intensity of the microscopic pictures was analyzed using ImageJ for immunofluorescence quantification. Four microscopic pictures were taken per section. The average background intensity of 3 measurements was subtracted from each image. A total of 15 cells per section were selected using the freehand drawing tool, and the area and integrated density were measured. The following formula was used to obtain the fluorescence intensity: fluorescence per pixel $=$ total integrated density $/$ total area.

\section{Data processing and statistical analysis}

The raw sequencing data was aligned to the $\mathrm{mm} 10$ genome using the STAR v2.4.0 h aligner with the default settings. Duplicate reads were marked using the MarkDuplicates tool in picard-tools-1.8.4 and removed. The uniquely aligned reads were then counted against Gencode's GRCm38.p3 GTF annotations using htseq-count in the intersection-strict mode. Final read count files were generated with HTSeq-count version 0.6.1p1. Data analysis of mapped counts was performed in $\mathrm{R} 3.2 .3$ using the DESeq2 package (version 1.10) [27]. Samples with less than 6000 genes with at least 5 mapped reads were excluded from analysis $(n=0)$. For unsupervised clustering, sample read counts were normalized using the regularized logarithm transformation method, which is similar to $\log _{2}$ transformation for genes with high counts and shrinks together the values for low count genes [27]. The regularized logarithm (rlog) values were used to plot heatmaps using the gplots (version 2.17) heatmap.2 function in R. Unsupervised clustering was performed based on the top 750 most variable genes between samples. Differential expression analysis was performed in DESeq2, and only two-sided Benjamini and Hochberg multiple testing adjusted $p$ values are reported in this manuscript. The level of significance used is $<0.05$ Benjamini and Hochberg multiple testing adjusted $p$ value. Error bars display mean \pm standard error of the mean (SEM). The "n" represents three individual mice for the EV-GFP ${ }^{\text {pos }}$ microglia and GFP $^{\text {neg }}$ tumor and control microglia experiments.

For analysis of specific gene sets, the microglial sensome was extracted from Hickman et al. ${ }^{8}$. The human sensome was derived in a similar manner as the mouse sensome (manuscript in preparation). The IL6/STAT3 and TGF- $\beta$ sets were extracted from the gene set enrichment analysis (GSEA) hallmark collection [28]. The IL4, IL10, and IFNY sets were calculated from the Xue et al. [29] study by extracting the 150 highest upregulated genes compared to baseline. For the IL6/STAT3, TGF- $\beta$, IL4, IL10, and IFNY sets, human to mouse homolog conversions were performed using The Jackson Laboratory Human and Mouse Homology Report (accessed February 18th 2016) supplemented by manual curation. Venn diagrams were generated using the VennDiagram $R$ package (version 1.6.16) [30]. Principal component analysis (PCA) was performed by utilization of the DESeq2's built-in PCA function using the default settings. Final bar graph, dotplots, PCA, and MA plots were generated in GraphPad Prism (version 5.0c and 7.02).

\section{Statistical analysis of human glioblastoma macrophage/ microglia data}

Data on bulk human glioblastoma macrophages/microglia was downloaded from the NCBI Gene Expression Omnibus (GSE80338) as deposited by Szulzewsky et al. [31]. For comparative expression analysis, only samples from glioblastoma patients $(n=8)$ and postmortem controls $(n=5)$ were used. Samples with less than 6000 genes with at least 5 mapped reads were excluded from analysis $(n=0)$. The sample-to-sample heatmap was generated using the Pheatmap $\mathrm{R}$ package version 1.08 and using the Eucladian distance between samples.

Single-cell glioblastoma microglia data was extracted from http://www.gbmseq.org/ and described and published by Darmanis et al. [32]. Similar to the original publication, every cell in the myeloid clusters were allocated to the subgroup of either macrophage or microglial origin, based on the mean expression of macrophage (CRIP1, S100A8, S100A9, ANXA1, and CD14) or microglia (TMEM119, P2RY12, GPR34, OLFML3, SCL2A5, SALL1, and ADORA3) markers [32]. For every microglia cell, t-distributed stochastic neighbor embedding (tSNE) mapping was performed based on the published coordinates for every cell in the dataset using ggplot2 version 2.2.1. The normalized read counts and 
differential expression data were extracted for every microglial cell comparing glioblastoma core cells to peripheral cells using DESeq2 similar as described above.

\section{Results \\ Diffuse microglia, monocytes, and macrophage infiltration in glioblastoma}

To identify immune cells that had taken up tumor-derived GFP and thus interacted with the tumor (Fig. 1a), we implanted syngeneic mouse glioblastoma cells, GL261.BpalmGFP, or carrier medium in adult C57BL6.CCR2 $2^{\mathrm{RFP} / W T}$ mice that express red fluorescent protein (RFP) under the CCR2 promoter in peripheral blood monocytes and monocyte-derived macrophages, but not in microglia [21]. Four weeks following implantation, the mice were euthanized, and the brains used either for immunofluorescent staining of brain sections or for FACS of brain cells. Using this model, tumor cells express GFP, microglia are labeled with antibodies to IBA-1, and recruited monocytes and macrophages express RFP (Fig. 1b, c). Microglia, monocytes, and macrophages that are closely interacting with glioblastoma cells are positive for IBA-1 and RFP respectively (Fig. 1b). Confocal microscopy and 3-dimensional reconstruction confirmed that GFP is found inside these IBA-1 ${ }^{\text {pos }}$ microglia (Fig. 1c).

For FACS, we generated highly enriched microglia, monocyte, and macrophage populations from the brains of tumor-bearing and control mice using an established protocol for cell dissociation, isolation, and analysis [26, 33, 34]. Microglia were sorted based on levels of CD11b and CD45 (Fig. 1d). Monocytes and macrophages were separated by additional staining for F4/80 and LY6C, as well as by expression of CCR2-RFP (not shown) [21, 35, 36]. The cells were isolated from brains injected with only carrier fluid (control), GL261, or GL261.BpalmGFP tumor cells. Microglia, monocytes, and macrophages were then sorted based on their level of GFP fluorescence to separate cells that had taken up tumor-derived membranous material from those that had not (Fig. 1d). The GFP cutoff was determined by comparing the relative GFP intensity detected in our target cell subsets isolated from brains injected with GL261 wildtype (no GFP) to brains injected with GL261.BpalmGFP (Fig. 1d). By separately analyzing the tumor area, as well as the remaining ipsilateral and contralateral sides of the brain (Fig. 1e), we found that EV-GFP positive $\left(G^{2} P^{\text {pos }}\right)$ microglia, monocytes, and macrophages were only present within and immediately adjacent to the tumor, confirming that the GFP $^{\text {pos }}$ cells are closely associated with tumor cells (Fig. 1f). Total RNA was isolated, and sequencing libraries were made using SMARTer Ultra Low Input RNA Kit. Sequencing was done using an Illumina NextSeq, and bioinformatic analysis was performed using DESeq2 in R [27].
An interactive online tool for analysis of gene expression in microglia, monocytes, and macrophages in glioblastoma

Using this approach, we generated a comprehensive dataset with comparative transcriptomes of control microglia (carrier-injected mice), GFP ${ }^{\text {pos }}$ glioblastomainteracting microglia (EV-GFP ${ }^{\text {pos }}$ microglia), and glioblastoma $\mathrm{GFP}^{\text {neg }}$ microglia. To facilitate analysis of these datasets, we developed an interactive online tool with additional functional and pathway information linked to every gene. The microglia dataset is accessible at http:// www.glioma-microglia.com.

To illustrate the usefulness of our dataset, we performed an in-depth analysis of the microglia data and include it in this manuscript. Normalized expression counts and differential expression data available for all genes passing quality metrics are available in Supplementary Table S1a. When analyzing the highest expressed genes in the control, GFP ${ }^{\text {neg }}$ microglia, and EV-GFP ${ }^{\text {pos }}$ microglia, multiple established microglia genes such as $C x 3 c r 1, \operatorname{HexB}$, and P2ry12 were among the most highly expressed [26, 34] (Supplementary Table S1A). To determine if differential RNAseq expression correlated with differential protein levels, we performed immunofluorescent staining for IBA-1, CD74, and ARG1 comparing the level in microglia from control brains versus tumor-bearing brains and the gene expression level of these genes in the differently sorted microglia populations. Similar levels of IBA-1 protein and RNA levels were detected comparing control versus tumor microglia (Supplementary Fig. 1A-C). In parallel, elevated levels of $C d 74$ and Arg1 RNA in tumor microglia was also detected at the protein expression level of CD74 and ARG1 protein (Supplementary Fig. 1A-C). Overall, these results showed a strong correlation between RNA and protein levels.

\section{EV-GFP ${ }^{\text {pos }}$ microglia represent the most influenced tumor- associated microglia}

Unsupervised clustering of the top 750 most differentially changed genes showed a clear separation of microglia from control versus tumor-bearing mice, as well as a separation based on GFP status of microglia in tumorbearing mice (Fig. 2a). When plotting levels of expression for all genes, comparing expression of $G P^{\text {neg }}$ microglia and EV-GFP ${ }^{\text {pos }}$ microglia versus control microglia, we found that for most genes, differential expression was stronger for EV-GFP ${ }^{\text {pos }}$ microglia than GFP $^{\text {neg }}$ versus control microglia (Fig. 2b). Expression of 380 genes was significantly changed in both $G P^{\text {neg }}$ microglia and EV-GFP ${ }^{\mathrm{pos}}$ microglia compared to control microglia. In contrast, 2242 genes were significantly changed only in EV-GFP ${ }^{\text {pos }}$ microglia (but not in GFP ${ }^{\text {neg }}$ microglia) compared to control (Fig. 2b). Comparison of 


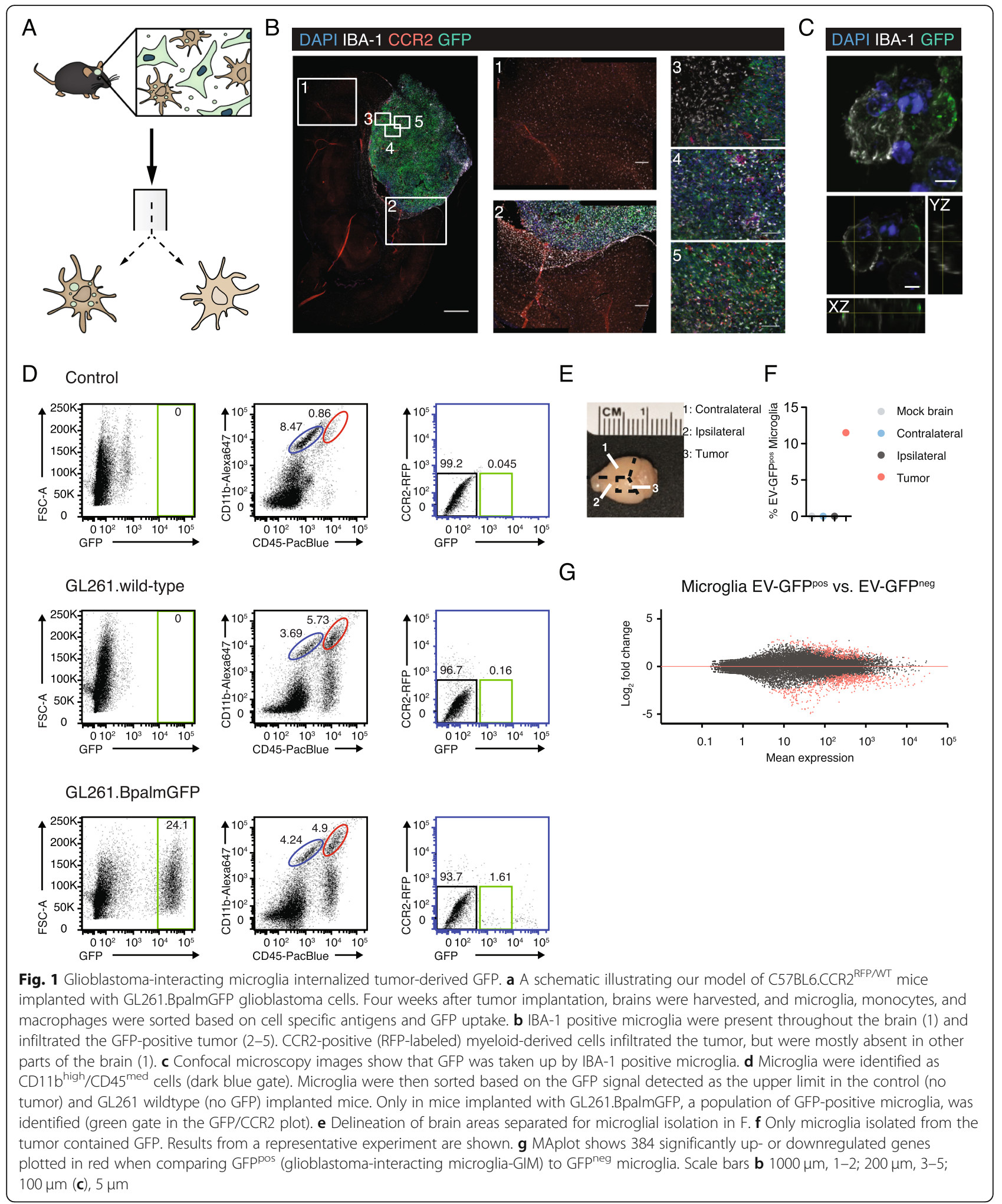

differential expression between EV-GFP ${ }^{\text {pos }}$ microglia versus GFP $^{\text {neg }}$ or control microglia showed that most genes that are significantly altered in $\mathrm{GFP}^{\mathrm{pos}}$ versus $\mathrm{GFP}^{\text {neg }}$ microglia are also significantly changed in EV-GFP ${ }^{\text {pos }}$ microglia versus control microglia (Fig. 2c). Comparing GFP $^{\text {neg }}$ microglia to either EV-GFP ${ }^{\text {pos }}$ microglia or control microglia confirmed these results (Fig. 2d). Evaluation of overlap between the top 750 genes expressed by 


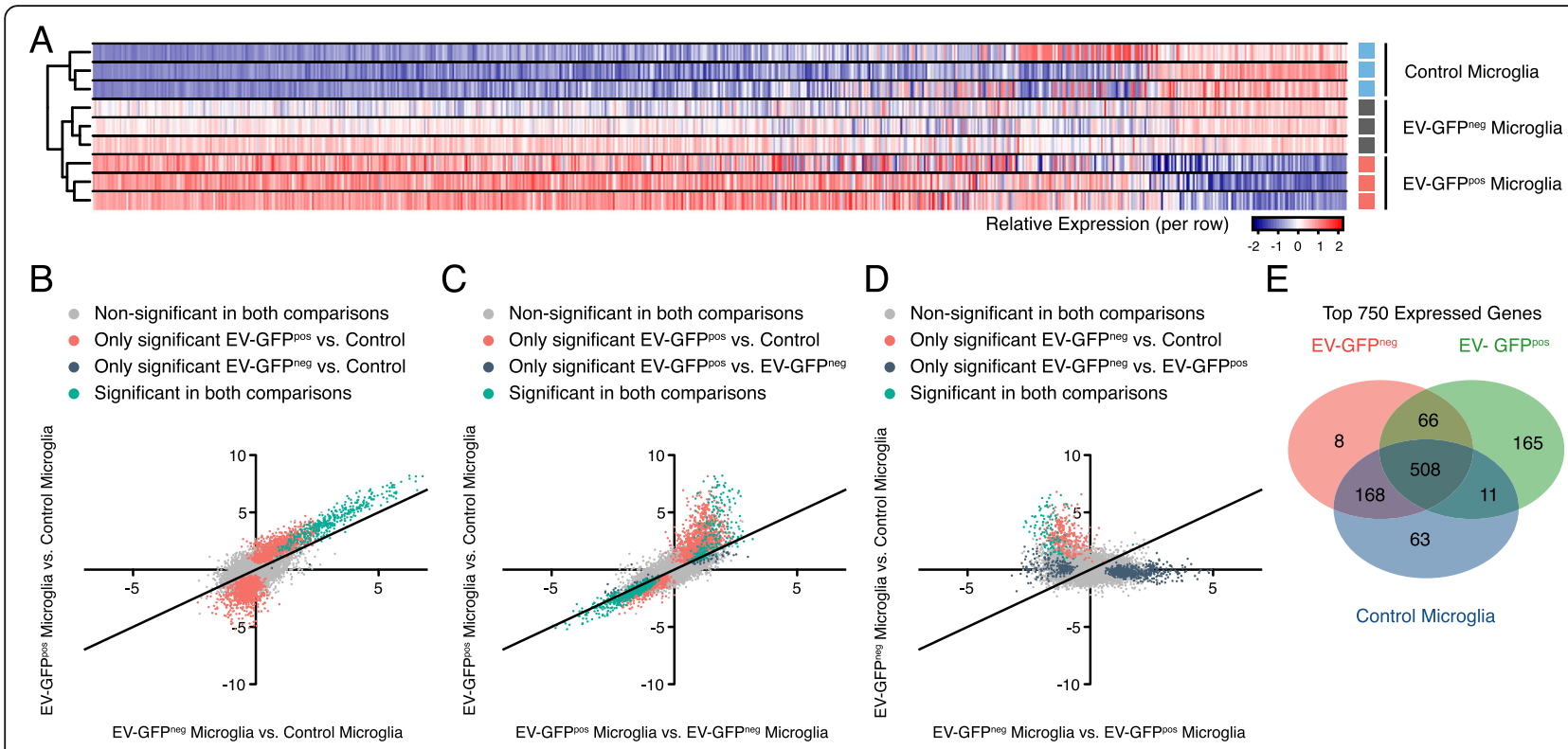

Fig. 2 RNA Expression changes are most pronounced in EV-GFPpos microglia compared to GFPneg microglia. a In unsupervised clustering of the top 750 most variable genes, microglia cluster together based on tumor status and GFP uptake status. b Comparative analysis of differential expression levels of EV-GFPpos microglia and GFPneg microglia compared to control microglia showed 380 shared significantly upregulated genes (green). Overall, the differential expression was higher for EV-GFPPos microglia. These microglia expressed 1426 significantly upregulated and 1196 downregulated genes (red). c Most genes significantly changed between EV-GFPpos microglia and GFPneg microglia and were also significantly altered in EV-GFPpos microglia compared to control. $\mathbf{d}$ These patterns were confirmed in the comparisons of GFP ${ }^{\text {neg }}$ to either GFPpos or control. e Venn diagram showed overlap between top 750 expressed genes. GFPneg tumor microglia shared most genes with control microglia and EVGFP ${ }^{\text {Dos }}$ microglia. This confirmed that EV-GFP ${ }^{\text {pos }}$ microglia represent the most altered tumor-associated phenotype

the three sets of microglia showed most uniquely expressed genes in either control or EV-GFP ${ }^{\text {pos }}$ microglia, with $\mathrm{GFP}^{\text {neg }}$ microglia being in-between (Fig. 2e). This analysis indicates that EV-GFP ${ }^{\text {pos }}$ microglia represent a subset of microglial cells that are the most influenced by the tumor.

\section{Cytokine pathways}

The concept that microglia are activated to either an "M1" (INFY stimulated) or "M2" (IL4 stimulated) state is actively debated in the current literature [37, 38]. We analyzed our dataset to determine if glioblastoma affects microglial cytokine pathways in vivo. We focused on the four pathways regulated by IL4, IL10, IL6/STAT3, and INFY (Supplementary Figure 2). Overall, analysis of the cytokine signatures in our dataset shows that several tumor-supportive genes belonging to multiple cytokine-related pathways are upregulated in glioblastoma-associated microglia in vivo indicating a more complicated profile than the binary M1/M2 classification. Correlation between gene expression, protein levels, and microglial functions should therefore be performed to de-

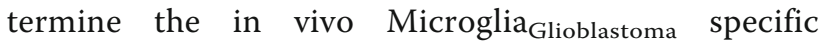
phenotype.

\section{Effect of glioblastoma cells on genes involved in key microglial functions}

Microglia are involved in brain development, aging, response to injury, and various pathological conditions [38-40]. Microglia have three major functions. First, they continuously survey their milieu to sense changes in their environment. Second, they help protect the brain from invading pathogens and noxious stimuli [41]. Third, they promote homeostasis and synaptic remodeling in development and learning [38, 42]. Microglia express clusters of genes that allow them to perform their different functions and have a number of distinct transcriptomic signatures, which vary with the physiological and/or pathological state of the brain [26, 34]. The homeostatic functions of microglia and expression of genes involved in these functions are regulated by TGF$\beta[43,44]$. To determine the effects of glioblastoma cells on the three essential microglial functions, we mined our dataset for genes and pathways involved in each of these functions.

\section{Homeostasis}

TGF- $\beta$ regulates the microglial homeostatic phenotype $[44,45]$. We found that only in $\mathrm{GFP}^{\mathrm{pos}}$ microglia both $T g f-\beta 1$ and the Tfg- $\beta$ receptor $1(T g f-\beta r 1)$ are significantly downregulated compared to control microglia 
( $\log _{2}$ fold-change -1.00 and -2.11 , respectively) (Supplementary Table S1B). A global view of the TGF- $\beta$ pathway revealed that $64.2 \%$ of TGF- $\beta$ genes are downregulated when comparing EV-GFP ${ }^{\mathrm{pos}}$ microglia to control microglia (Fig. 3a and Supplementary Table S1B). Smad3, one of the key downstream effectors in the TGF- $\beta$ pathway, is also significantly downregulated in EV-GFP ${ }^{\text {pos }}$ microglia ( $\log _{2}$ fold-change - 2.10) (Fig. 3a and Supplementary Table S1A). Overall, these data imply that TGF- $\beta$ signaling is downregulated in EVGFP $^{\text {pos }}$ microglia suggesting a disruption in microglial homeostasis.

\section{Host defense}

The third important microglial function is host defense against viral, bacterial, fungal, and parasitic infections, but also against tumor cells [46]. We mined our dataset for microglial genes involved in this function. Interactions of the programmed cell death 1 receptor (PD1) on activated $\mathrm{T}$ cells with its ligands programmed death ligand 1 and 2 (PD-L1 and 2) maintain immunologic tolerance through the suppression of auto-reactive $\mathrm{T}$ cells [47]. PD-L1 and PD-L2 are expressed on antigenpresenting cells, as well as on tumor cells including glioblastoma [48, 49]. As expected, very little Pd1 RNA was expressed in microglia as it is usually expressed on $\mathrm{T}$ cells [50]. However, increased expression of $P d-l 1$ and $\mathrm{Pd}-\mathrm{l} 2$ transcripts was higher in EV-GFP ${ }^{\text {os }}$ microglia as compared to $\mathrm{GFP}^{\text {neg }}$ microglia with both being significantly higher than for control microglia (Fig. 3g). These data identify another pathway by which glioblastoma can possibly evade the immune system, by altering microglia to suppress $\mathrm{T}$ cell activation through modulation of $\mathrm{T}$ cell immune checkpoints. This finding gains added importance as PD1/PD-L1 directed immune checkpoint therapy is being used against a number of peripheral tumors [51].

\section{Sensing}

The ability to sense changes in the cellular environment in the brain is a major microglial function that allows these cells to adapt to and influence the changing milieu $[52,53]$. The armamentarium of 100 genes that allow microglia to perform such functions is termed the sensome [26] (Supplementary Table S1A). These include pattern recognition receptors $(25 \%)$, receptors involved in cell-cell interaction (10\%), chemoattractant and chemokine receptors $(10 \%)$, cytokine receptors $(10 \%)$, Fc receptors $(7 \%)$, purinergic receptors $(8 \%)$, receptors for extracellular matrix (ECM) proteins (6\%), other receptors or transporters (13\%), and potential sensome proteins with no known ligands (11\%) [26]. When analyzing expression levels of genes involved in microglial sensing, we identified overall downregulation of the sensing capacity in glioblastoma-interacting microglia (Fig. 3b).

Sensome transcripts that were downregulated in EV$\mathrm{GFP}^{\text {pos }}$ microglia compared to $\mathrm{GFP}^{\text {neg }}$ and control microglia can be divided into three groups. Group one includes transcripts encoding proteins that directly mediate microglia-glioblastoma cellular interactions. Indeed, sialic acid-binding immunoglobulin-like lectin- $\mathrm{H}$ (Siglech) is a CD33-related Siglec that is a microglial sensor of glioblastoma cells [54]. Siglech is significantly downregulated in EV-GFP ${ }^{\text {pos }}$ microglia compared to GFP $^{\text {neg }}$ and control microglia ( $\log _{2}$ fold-change - 1.84 and -1.97 , respectively) (Fig. 3c). Interestingly, $C d 33$ is also significantly downregulated in $\mathrm{GFP}^{\mathrm{pos}}$ compared to GFP $^{\text {neg }}$ and control microglia ( $\log _{2}$ fold-change - 1.62 and -1.72 , respectively) (Fig. 3c). It is not known if CD33, like Siglech, is also a sensor of glioblastoma cells. Another microglial receptor that is capable of sensing lysophosphatidylserine exposed on glioblastoma cells is GPR34 [55, 56]. Similar to Siglech, Gpr34 a gene known to directly sense ligands expressed in glioblastoma cells is downregulated in EV-GFP ${ }^{\text {pos }}$ microglia compared to $\mathrm{GFP}^{\text {neg }}$ and control microglia ( $\log _{2}$ fold-change - 1.96 and - 2.37, respectively) (Supplementary Table S1A). These data indicate that EV-GFP ${ }^{\text {pos }}$ microglia, but not other microglia in the same tumor-bearing brain, have reduced expression of at least two transcripts, encoding the proteins SIGLECH and GPR34, known to directly sense ligands expressed on glioblastoma cells.

A second group of transcripts that is downregulated in EV-GFP ${ }^{\text {pos }}$ microglia, but not in GFP $^{\text {neg }}$ microglia, includes those encoding proteins that sense metabolic products potentially released by glioblastoma cells. These transcripts include Gpr183, Adora3, Il6Ra, Cx3cr1, P2ry12, P2ry13, Csf1r, and Csf3r (Fig. 3d). GPR183 is a sensor for oxysterols, which are released by glioblastoma cells and play a role in recruitment of immune cells [57]. ADORA3 is a sensor for adenosine that is released by glioblastoma cell ectonucleotidases. Adenosine promotes tumor growth, can activate toll-like receptors (TLRs), and induces microglial responses via an ADORA3-dependent mechanism [58]. IL6Ra is a receptor for IL6, with elevated levels of IL6 in glioblastomas associated with poor survival in patients [59]. Expression of the fractalkine receptor CX3CR1 was also decreased, and loss of CX3CR1 has been shown to promote glioblastomagenesis [60]. P2RY12 and P2RY13purinergic receptors for ATP, which is an important signaling molecule in the CNS, are both down (Fig. 3d). This could promote tumor growth by two different pathways. First, necrosis, one of the hallmarks of glioblastoma, liberates nucleotides into the extracellular milieu. These nucleotides are hydrolyzed very slowly by glioblastomas and induce neuronal cell death and 


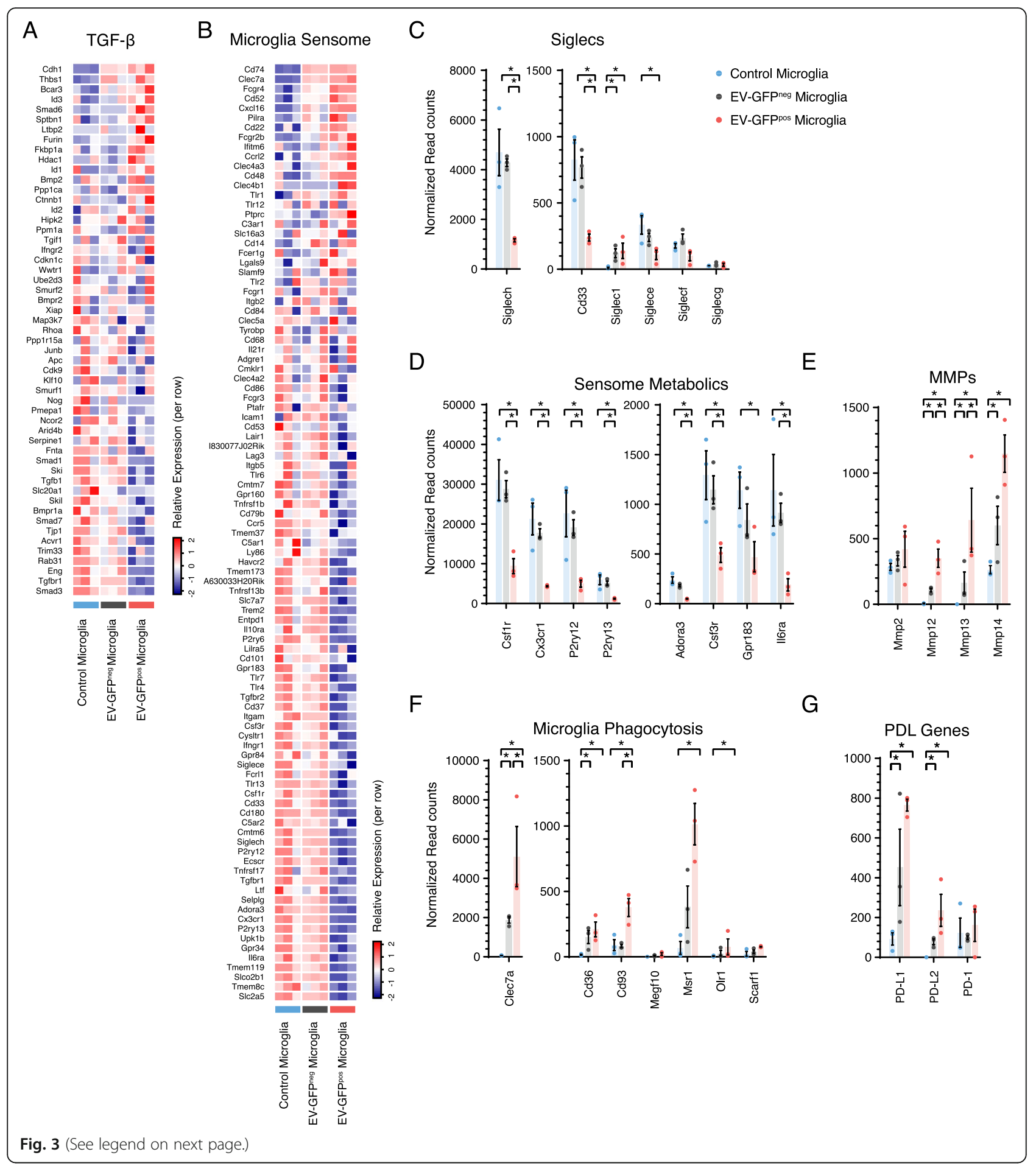




\section{(See figure on previous page.)}

Fig. 3 Glioblastoma microglia have a downregulated homeostatic TGF- $\beta$ pathway, tumor-derived danger signal sensing capacity, and disrupted host defense. a TGF- $\beta$ is the key regulator for microglial homeostasis. In GIM, Tgfb 1 and downstream signaling genes including Smad3 are significantly downregulated, indicating a disruption of homeostatic functions. b EV-GFP ${ }^{\text {pos }}$ microglia showed significantly reduced levels of 57\% of microglial sensome genes compared to GFP neg, indicating reduced capability of sensing of tumor cells and tumor-derived danger signals in EV-GFPpos microglia. c Normalized read counts of Siglecs, involved in direct glioblastoma-microglial cellular interactions, showed significant downregulation of Cd33, Siglece, and Siglech in (GFP ${ }^{\text {pos }) ~ G I M, ~ w h e r e a s ~ o n l y ~ S i g l e c 1 ~ w a s ~ u p r e g u l a t e d . ~ d ~ S e v e n ~ o u t ~ o f ~ e i g h t ~ s e n s o m e ~ g e n e s ~}$ involved in the sensing of metabolic signals were significantly downregulated in EV-GFPos microglia. e Matrix metalloproteinases (MMPs) were upregulated in GIM. Mmp12, Mmp13, and Mmp14 were significantly upregulated tumor supportive genes. f Genes involved in phagocytic activity in microglial cells were upregulated. Cd93 and Clec7a were significantly higher in EV-GFPpos microglia than GFPneg microglia. $\mathbf{g}$ Programmed death ligand 1 and $2(P d-/ 1$ and $P d-/ 2)$ were significantly upregulated in tumor-associated microglia. Asterisk $(*)$ indicates significant (multiple testing adjusted $p$ value $<0.05$ ) differential expression. Error bar represents the SEM, bar represents the mean, and dots display individual measurements (C-G $n=3$ )

glioblastoma proliferation [61]. Second, extracellular ATP activates microglial P2RY12 receptors that are utilized to trigger an acute inflammatory response in microglia via rapid CCL3 induction after ADP stimulation [62]. Therefore, downregulating microglial receptors for ATP could preserve the ability of the nucleotides to promote tumor growth, while reducing the ability of microglia to respond to the tumor, thereby further enhancing the tumor's advantage.

Of note, the overall expression pattern of all 100 sensome pathway genes showed differential gene expression in only $4 \%$ of sensome genes when comparing $\mathrm{GFP}^{\text {neg }}$ to control microglia (Fig. $3 \mathrm{~b}$ and Supplementary Table S1B). These genes (e.g., Cd74, Clec7a, Cxcl16, and Fcgr4) were all upregulated compared to control microglia. In contrast, we found significant changes in gene expression between EV-GFP ${ }^{\text {pos }}$ microglia versus GFP $^{\text {neg }}$ and control microglia in $57 \%$ of sensome transcripts. Remarkably, $48 \%$ of sensome genes were downregulated in GFP $^{\text {pos }}$ microglia and only $9 \%$ upregulated (Fig. 3b). This could indicate that the microglia infiltrated into glioblastoma are not able to sense the tumor. Overall, while further research is required to validate the exact impact of individual sensome genes on tumor growth, our results show that microglia dramatically change their expression profile in the presence of a tumor, reducing their capacity to sense changes in the (tumor) microenvironment.

\section{Pathways involved in tumor growth}

Since the expression of genes involved in the maintenance of homeostasis within EV-GFP ${ }^{\text {pos }}$ microglia are disrupted, we investigated the effects of this disruption on three pathways that maintain brain homeostasis and affect tumor growth. The role of microglia in maintaining brain homeostasis includes debris breakdown and removal by matrix metalloproteases (MMPs) [26]. MMP enzymes could also play an important role in promoting tumor growth by making space for tumor cells to migrate, invade, and proliferate $[3,9,10]$. In glioblastoma, MMP2 serves as an important MMP to degrade the extracellular matrix (ECM) subsequently enabling the invasive properties of glioblastoma [63]. MMP2 is secreted by glioblastoma cells in a pro-form (pro-MMP2) which needs to be cleaved by Mmp14 (MT1-MMP) to be active $[9,10]$. Tumor microglial cells are an important source of MMP14 [9, 10]. Previously, we showed that Mmp14 levels are increased in glioblastoma-associated microglia in vitro [17]. Mmp14 was among the three Mmps (Mmp12, Mmp13, and Mmp14) that were significantly upregulated in EV-GFP ${ }^{\text {pos }}$ microglia and to a lesser extent in $\mathrm{GFP}^{\text {neg }}$ microglia (Fig. 3e). These data indicate that glioblastoma alters microglial gene expression patterns in a manner that could favor tumor spread and migration by clearing debris and digesting the ECM in the tumor microenvironment.

In addition to changes in $M m p s$, we also found that glioblastoma was associated with an increased expression of mRNAs encoding microglial phagocytic receptors-Cd93, Msr1, Cd36, Olr1, Megf10, Clec7a, and Scarf1 (Fig. 3f). The roles of these phagocytic receptors in promoting debris clearance and subsequent tumor growth have not yet been investigated. However, since these receptors promote clearance of apoptotic cells [64], it is plausible that these receptors, in conjunction with MMPs, promote the phagocytic clearance of debris in the tumor environment further facilitating tumor spread.

\section{Microglial uptake of EVs is associated with decreased sensome expression}

To explore the relationship between microglial uptake of glioblastoma derived EVs and the expression of sensome genes, we evaluated RNA expression by microglia isolated from control (non-tumor bearing) C57BL6.CCR2 ${ }^{\text {RFP/WT }}$ mice injected with carrier fluid or with EVs isolated from GL261.BpalmGFP cells. EVs were isolated using standard step-wise (ultra)centrifugation (Fig. 4a) and as expected, the isolated EVs were within the $80-400-\mathrm{nm}$ size range (Fig. 4b) expressing the EV associated proteins ALIX, TSG101, and Flotillin-1 as well as GFP (Fig. 4c). Sixteen hours after EV injection, 
A

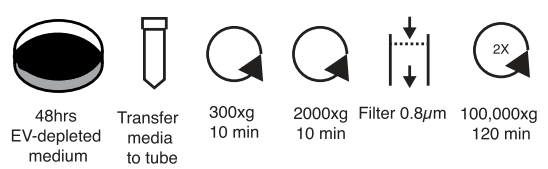

B

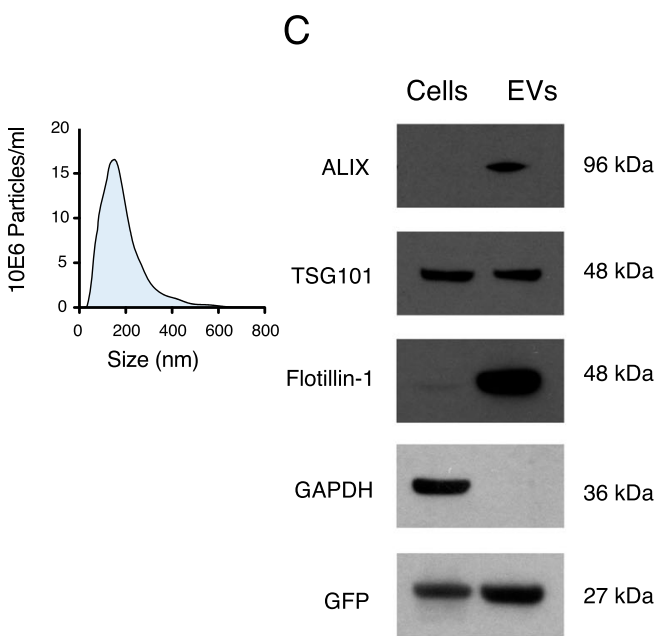

D

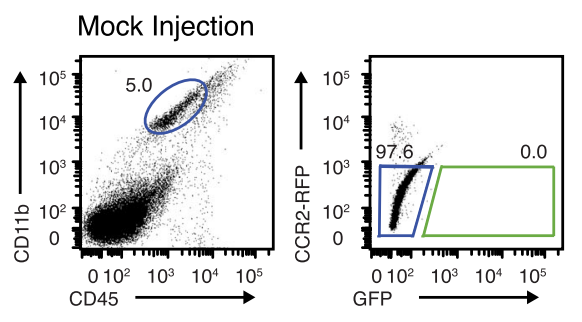

GL261.BpalmGFP EV Injection

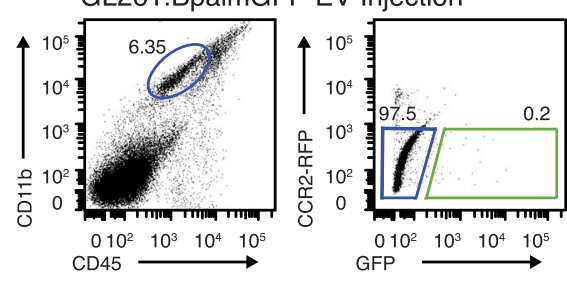

E

Microglia Sensome

Cd74
clec7a
Cont

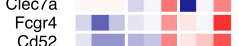

Cxc116

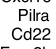

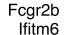

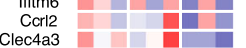

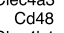

Clect41
Tr1
Trr12
Cest

Ptprc
C3ar1

C3ar1
Slc163
Cd14

Cd14
Fcer19
Catg

Lgals9

Slamig

Trr2
Fcgr1
Itgh2

$\operatorname{ltgb2} 2$
$\mathrm{C} d 84$

Co84
Clec5a
Cos

Tyrobp
Cd68

Cd68
II21r

Adgre1

Cmkl1
Clecta2
Chas

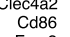

$\underset{\text { Fogrs }}{\mathrm{CAB}}$

Pcors
Ptatr
Catr

Icam1

1830077J02Riik

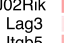

Lag3
Itgb5
TIr6

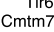

Gpr160

Tnfrsf1b
Cd79b

Ca79b
Cors
Cist

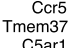

C5ar1
Ly86

Ly86
Havcr2
Tmem173

Them173

Slc7a7

Trem2
Entrod1
The

Entpd
IIIOra
PLI

P2ry6
Lilra5

Cliras
Cd101

Gpr183
TIr7

Gpr183
TIr7
Tir4
Tgfbr2
Ctat

$\mathrm{Tg} f b r 2$
$\mathrm{Cd} 37$

Itgam
$c s 3 r$

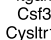

Cystrtr
Inngrt
Shat

Gipr84

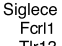

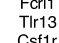

Cd33

Cd180
C5ar2

Cmtm
Siglech
P2ry12

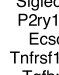

Tntrst

Selp
Adoras

Selpll
Adora3
Cx3cr
P2ry13

Prry13
Upk1b
Gpr34

Gpr34
I16ra
Tmem119
Stion

Tmem119
SIco2b1
Slc2a5

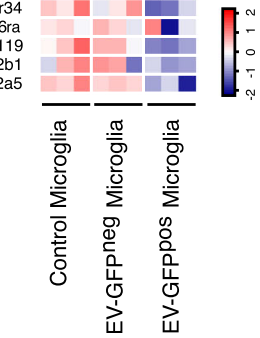

Fig. 4 (See legend on next page.) 
(See figure on previous page.)

Fig. 4 Uptake of intracranially injected glioma-derived fluorescent EVs is associated with a decrease in microglial sensing capability. a Schematic overview of EV isolation from glioma cells in culture using differential centrifugation. $\mathbf{b}$ Size distribution analysis using NTA of isolated EVs shows small and larger vesicles present in the EV preparation. c Western blot analysis shows GFP present in cells and EV, and extracellular vesicles markers (ALIX, TSG101 and Flotillin-1) enriched in vesicles lysate and GAPDH is detected in cellular lysate only. $\mathbf{d}$ Microglia were identified as CD1 $1 b^{\text {high }} / C D 45^{\text {med }}$ cells (blue gate). Microglia were then sorted based on the GFP signal detected as the upper limit in control. In mice injected with GL261.BpalmGFP EVs, a population of GFP-positive microglia was identified (green gate in the GFP/RFP plot). e Heatmap of sensome genes ordered top to bottom by highest up- to downregulated for mouse EV-GFPpos tumor microglia compared to wildtype (same order as Fig. 3b). Similar patterns are observed for genes up- and downregulated compared to the mouse tumor-derived profile. Asterisk $(*)$ indicates significant (multiple testing adjusted $p$ value $<0.05$ ) differential expression. Error bar represents the SEM, bar represents the mean, and dots display individual measurements $(n=3)$

microglia were isolated based on EV uptake and their transcriptomes analyzed by RNASeq (Fig. 4d). Similar to the results from EV-GFP ${ }^{\text {pos }}$ microglia isolated from tumor bearing brains, overall downregulation of the microglia sensome genes was observed in microglia that took GFP-EVs injected into the brain (Suppl. Table S2 and Fig. 4e). It is possible that some of the changes observed in EV-GFP ${ }^{\text {pos }}$ microglia did not reach significance because the number of EVs added and time point of analyses may bias the result. These data show parallels between tumor microglia and microglia isolated after $\mathrm{EV}$-injection and open the door for further investigation of specific EV contents that may induce the changes observed.

\section{Human glioblastoma-associated microglia have a reduced sensing capacity}

To determine if changes in gene expression in human glioblastoma-associated microglia are similar to those observed in mouse microglia, we analyzed two existing published datasets of human microglia. These datasets contain bulk RNA sequencing results comparing postmortem brains (controls) to $\mathrm{CD} 11 \mathrm{~b}^{\text {pos }}$ macrophage/ microglia isolated from glioblastoma samples (GEO Accession GSE80338) [31] and single-cell RNA sequencing data comparing microglia isolated from either the core or the periphery of the glioblastoma tumor mass (data from http://www.gbmseq.org/) described and published by Darmanis et al. [32]. As expected, the control and glioblastoma-associated cells cluster separately with some heterogeneity within the glioblastoma samples (Fig. 5a). Similar to our mouse samples, 32\% of human microglial sensome genes were downregulated, and only $12 \%$ were upregulated in human glioblastoma microglial cells compared to control, paralleling our data obtained from mice (Fig. 5b).

We then assessed if these results could be confirmed using published single-cell microglia data from human patients with glioblastoma. These data were obtained from microglia isolated either from the core of a glioblastoma tumor or the periphery [32]. Since microglia within the tumor mass are more likely to interact directly with tumor cells than microglia from the periphery of the tumor, we hypothesized that microglia from the human tumor core will most likely resemble mouse GIM and will have similar glioblastoma-induced RNA expression to mouse GFP ${ }^{\text {pos }}$ microglia. To separate microglia from macrophages in the dataset, we used the expression levels of TMEM119, P2RY12, GPR34, OLFML3, SCL2A5, SALL1, and ADORA3 as microglial markers and CRIP1, S100A8, S100A9, ANXA1, and CD14 as macrophage markers [26]. By focusing on the identified microglia only, we could see clear separation of microglial cells isolated from the core or periphery of the tumor (Fig. 5c). Similar to our results from mice, the microglia isolated from the core of human glioblastoma have a reduced sensing capacity with significantly reduced expression of $48 \%$ of sensome genes versus only 15\% upregulation (Fig. 5d).

Taken together, these data identify reduced expression of microglia sensing genes in glioblastoma microglia suggesting reduced sensing capacity in these cells (Fig. 5e).

\section{Discussion}

Glioblastomas are the most aggressive malignant brain tumors leading invariably to death. To date, no effective therapy has been found for this devastating disease. These tumors are heavily infiltrated with innate immune cells including resident brain microglia. Yet, despite such a large immune cell presence, glioblastomas continue to grow and are thought to co-opt the innate immune system of the host to promote tumor spread [3]. To determine how glioblastoma affects the innate immune system, we analyzed the gene expression profile of microglia in a mouse model of this tumor using RNA sequencing. By using glioblastoma cells with fluorescently labeled membranes, we could identify and separate microglial cells closely associated with the tumor by their uptake of tumor-derived fluorescent membranes/ membrane particles including EVs (EV-GFP ${ }^{\text {pos }}$ ) from those EV-GFP ${ }^{\text {neg }}$ microglia that were further away from the tumor. We compared EV-GFP ${ }^{\text {pos }}$ and EV-GFP ${ }^{\text {neg }}$ microglia with each other and with microglia isolated from normal brains. Our data show that EV-GFP ${ }^{\text {pos }}$ glioblastoma microglia have a unique gene expression 


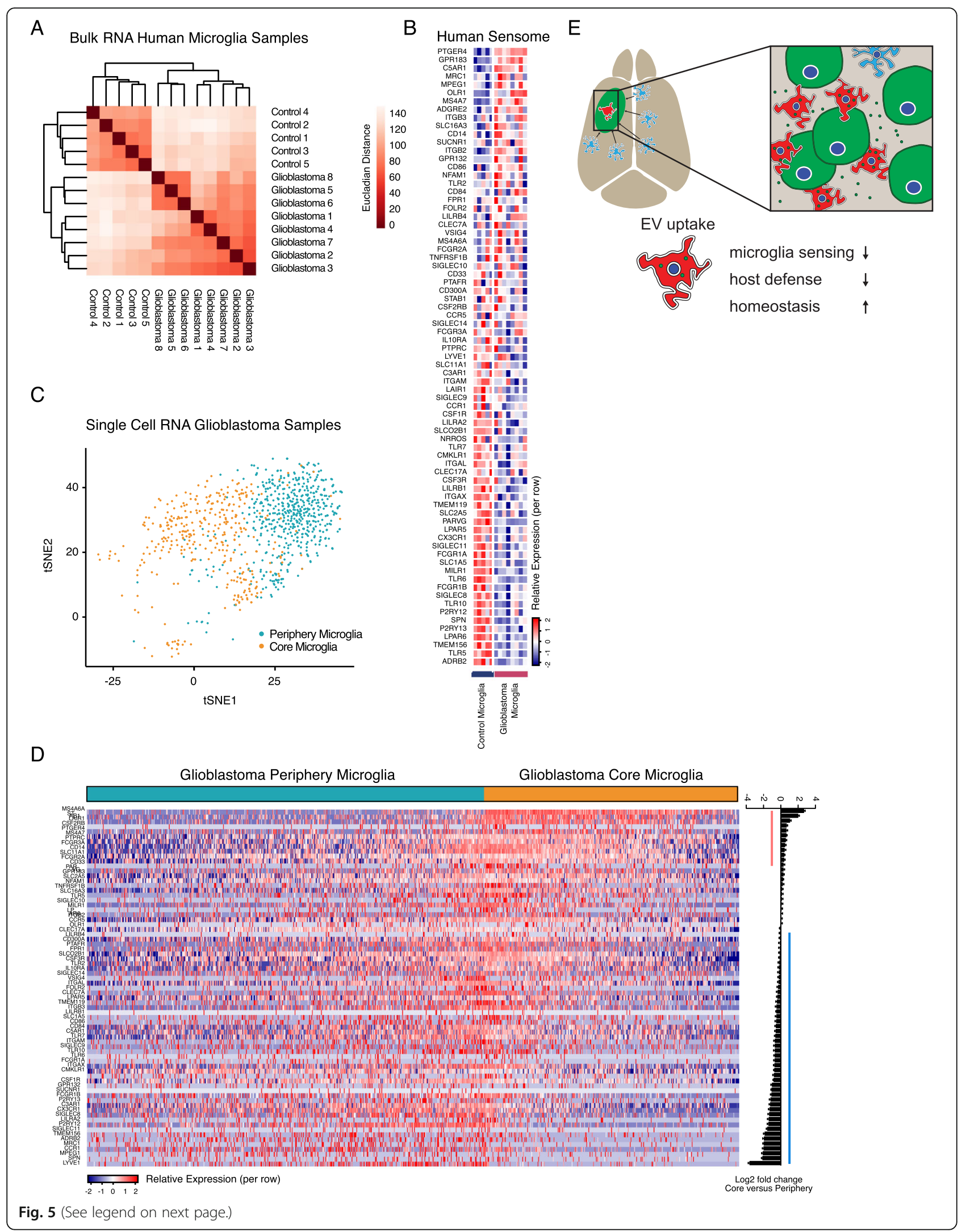


(See figure on previous page.)

Fig. 5 The Sensome is downregulated in human microglia from glioblastoma patients. a Analysis of published bulk RNAseq data from CD11B pos microglia harvested from postmortem human brains (control) or glioblastoma patients identifies differences based on sample group as well as heterogeneity between glioblastoma derived cells. b Glioblastoma microglia showed significantly reduced levels in 32\% of genes versus $12 \%$ upregulation, indicating reduced overall capability of sensing of tumor cells and tumor-derived danger signals in human glioblastoma microglia. Further analysis of published human glioblastoma single-cell microglia data identified similar results. c Expression levels of TMEM119, P2RY12, GPR34, OLFML3, SCL2A5, SALL1, and ADORA3 for microglia and CRIP1, S100A8, S100A9, ANXA1, and CD14 for macrophages were used to identify individual microglia and macrophages cells isolated at either the core or periphery of the glioblastoma mass. $\mathbf{d}$ At a single-cell level, $15 \%$ of genes are significantly upregulated (genes in red), and $48 \%$ of the human sensome genes are significantly downregulated (genes in blue) when comparing microglia at the core to microglia in the periphery of the glioblastoma mass again indicating reduced capability of sensing of tumor cells and tumor-derived danger signals in human glioblastoma microglia. e Schematic illustration showing the anti-tumor ability of microglia after EV uptake by simultaneous reduction of the sensing capacity and host defense as well as an increased homeostatic function. This pathway is ultimately required for glioblastoma growth. Asterisk $\left(^{*}\right)$ indicates significant (multiple testing adjusted $p$ value $<0.05$ ) differential expression. Error bar represents the SEM, bar represents the mean, and dots display individual measurements $(\mathbf{a}, \mathbf{b}$ : control $n=5, \mathrm{glioblastoma} n=8, \mathbf{C}, \mathbf{D}$ microglia core $n=365$, microglia periphery $n=574$ )

profile that distinguishes them from other microglia and that this glioblastoma-associated expression profile is more complex than the prior classification of M1 versus M2 states. Instead, we identified a disease-specific

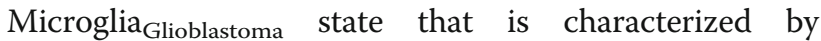
markers found in both M1 and M2 polarization states. This glioblastoma-associated expression profile defines a

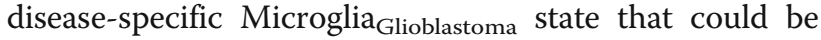
further subclassified based on proximity of the microglia

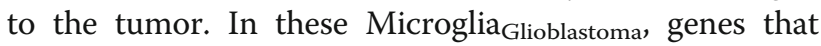
promote tumor killing are downregulated, whereas genes that promote tumor growth, invasion and immune suppression are upregulated.

We identified at least three pathways by which EVGFP $^{\text {pos }}$ microglia became less effective in combating the tumor and more geared towards promoting tumor growth. First, and most dramatically, we found that EVGFP $^{\text {pos }}$ microglia had reduced expression of genes involved in sensing tumor cells and tumor-derived cellular byproducts. A decreased ability to sense and recognize tumor cells makes these cells "hidden" from the immune system and therefore protected from anti-tumor immune activities. A second group of microglial transcripts altered by interaction with glioblastoma cells reflects a disarming of their usual anti-tumor functions. These include upregulation of PD-L1 and PD-L2 which help maintain immunologic tolerance by causing $\mathrm{T}$ cell exhaustion and ultimately reducing the tumor killing capacity of $\mathrm{T}$ cells [65]. We also found that microglial genes that suppress cytotoxic $\mathrm{T}$ cell activation and those in direct tumor killing, such as antimicrobial peptides are also suppressed $[66,67]$.

In contrast to reducing microglial tumor sensing and anti-tumor abilities, glioma cells enhance the capacity of microglia to promote tumor spread, by affecting genes that alter the extracellular milieu surrounding tumor cells. One of the hallmarks of glioblastoma is the presence of excessive debris and necrotic tissue, and clearing such necrotic material is important for tumor cell invasion and growth [68]. We found that microglia in the micro environs of tumors have increased expression of several phagocytic receptors, while either maintaining or increasing expression of extracellular matrix degrading enzymes. Clearing debris and necrotic tissue from the tumor milieu would boost the migratory capacity of tumor cells, one of the key characteristics of glioblastoma. These data indicate that glioblastoma-interacting microglia may help promote tumor growth and migration by clearing debris in the tumor microenvironment.

Our novel method of identifying microglia that have taken up tumor-derived EVs in vivo allows us to select microglia with which the tumor appears to have interacted directly with a physical exchange of membrane and cytoplasmic factors. Simultaneously, this could suggest that some of the gene expression changes observed are related to the uptake of EVs. In fact, when comparing microglia that took up glioma EVs in a non-tumor bearing brain to control microglia, we could detect similar gene expression changes as observed in EV-GFP pos tumor microglia. However, in the in vivo tumor model described here, all tumor lipid bilayers are GFP-positive and thus it is not clear whether all GFP ${ }^{\text {pos }}$ microglia have taken up EVs per se or may possibly have taken up tumor cell membrane debris. Other intercellular communication modes such as secreted molecules [69], exchange of molecules through gap junctions between cells [70], and cell connecting nano/microtubes may contribute to the observed effects as well. Glioma secreted cytokines (e.g., CSF-1, MCP-3, CX3CL1, SDF-1, and GM-CSF) that are especially known to be involved in the recruitment of microglial cells and could be responsible (in part) for the observed changes in gene expression, with EV-GFP uptake being a mere side-effect [3]

Glioblastomas are heterogeneous tumors at the interand intratumor level and they express gene patterns associated with mesenchymal, proneural, and classical subtypes [71]. We recognize that a single, highly clonal, 
murine glioma line may not recapitulate this heterogeneity. To address this issue, we analyzed existing datasets obtained from human patients with glioblastoma and found that these data support the conclusions obtained with our mouse model and reflect the true heterogeneity of human glioblastoma tumors, further asserting the validity of our analysis and its applicability to human disease.

For the sake of exploratory analysis and to increase the impact of our dataset, we established an online tool accessible at http://www.glioma-microglia.com that includes the microglia dataset. This webtool will facilitate the identification of additional genes associated with these tumors and are a useful tool for discovery.

\section{Conclusions}

Overall, our data open the door for future investigations to specifically identify how glioblastoma hijack the microglial immune response to promote tumor growth and will possibly help identify novel microglia-specific targets for therapy of this highly aggressive and so far, untreatable lethal disease. Our findings indicate that glioblastoma-associated microglia suppress the adaptive immune response to the tumor, have a reduced capacity to directly kill tumor cells, and promote tumor cell invasion and proliferation.

\section{Supplementary information}

Supplementary information accompanies this paper at https://doi.org/10. 1186/s12974-020-01797-2.

\section{Additional file 1: Table S1. $a, b$ all genes and subsets.}

Additional file 2: Figure S1. RNA levels correlated with protein levels in control and tumor-bearing brains. (A) The microglial marker lbal was equally expressed in control and tumor-associated microglia, whereas Cd74 and Arg1 expression was increased in tumor-associated microglia as measured by RNAseq. (B) Immunofluorescence staining of IBA1, CD74 and ARG1 in control and tumor-bearing mouse brains. (C) Quantification of immunofluorescent staining seen in (B) Fluorescent intensity was quantified per pixel within all identified cells. Tumor and control tissues were individually compared for each marker. IBA1, CD74 and ARG1 fluorescence quantification correlated with RNA data whereas Scale bars $100 \mu \mathrm{m}$, asterisk indicates multiple testing adjusted p-value $<0.05$, error bar represents SEM.

Additional file 3: Figure S2. IL4, IL 10, IL6 and IFNy pathways genes were upregulated in tumor-associated microglia. (A) The IL4 associated genes were mostly upregulated in tumor-associated microglia with increased expression in EV-GFP ${ }^{\text {pos }}$ microglia. The significantly upregulated genes in EV-GFP pos versus EV-GFP neg microglia included known tumor supportive genes such as Mmp12, Adam19 and Wnt5a. (B) IL10 related genes were upregulated in tumor microglia. Sod2, a tumor supportive gene, was among the genes significantly upregulated in EV-GFP pos microglia. (C) IL6 related genes were upregulated in tumor-associated microglia. Among the significantly upregulated IL6 genes is C I7 (MCP-3), a secreted chemokine involved in the attraction of microglia and macrophages to the tumor suggesting a tumor supportive infiltration loop. (D) Overall, increased expression of IFNy related genes was observed with the strongest expression in EV-GFPpos microglia. Among the significantly upregulated genes in EV-GFPpos microglia was Irf7, a key regulator of proinflammatory to anti-inflammatory switching in microglia.
Additional file 4: Table S2. EV injection data all genes and subsets.

\section{Abbreviations}

EVs: Extracellular vesicles; FACS: Fluorescence activated cell sorting; TGF$\beta$ : Transforming growth factor beta; DMEM: Dulbecco's modified Eagle's medium; FBS: Fetal bovine serum; TSG101: Tumor susceptibility gene 101; GAPDH: Glyceraldehyde 3-phosphate dehydrogenase; GFP: Green fluorescent protein; NGS: Normal goat serum; BSA: Bovine serum albumin;

ARG1: Arginase 1; CD74: Cluster of differentiation 74; IBA1: Ionized calcium binding adaptor molecule 1; CD45: Protein tyrosine phosphatase receptor type C (PTPRC); CD11b: Integrin alpha M (ITGAM); F4/80: EGF-like modulecontaining mucin-like hormone receptor-like 1 (EMR1); Ly6C: Lymphocyte antigen 6C; CCR2: C-C chemokine receptor type 2; PCA: Principle component analysis; MA: M (log ratio) and A (mean average); RFP: Red fluorescent protein; CX3cr1: CX3C chemokine receptor 1; HexB: Hexosaminidase subunit beta; Tgf- $\beta$ r1: Tfg- $\beta$ receptor 1; PD1: Programmed cell death 1 receptor; PDL1: Programmed death ligand 1; PD-L2: Programmed death ligand 2; Siglech: Sialic acid-binding immunoglobulin-like lectin-H; Cd33: Sialic acid binding Ig-like lectin 3 (Siglec-3); Gpr34: G-protein coupled receptor 34; Gpr183: G-protein coupled receptor 183; P2ry12: P2Y purinoceptor 12; P2ry13: P2Y purinoceptor 13; Csf1r: Colony stimulating factor 1 receptor; Csf3r: Colony stimulating factor 3 receptor; ADORA3: Adenosine $A_{3}$ receptor; TLR: Toll-like receptor; IL6Ra: Interleukin 6 receptor alpha; IL6: Interleukin 6; ATP: Adenosine triphosphate; CNS: Central nervous system; CCL3: Chemokine ligand 3; ADP: Adenosine diphosphate; Cxcl16: C-X-C Motif chemokine ligand 16; Fcgr4: Fc gamma receptor 4; MMP: Matrix metallopeptidase; Cd93: Cluster of differentiation 93; Msr1: Macrophage scavenger receptor 1; Cd36: Platelet glycoprotein 4; Olr1: Oxidized low-density lipoprotein receptor 1; Megf10: Multiple EGF like domains 10; Clec7a: C-type lectin domain containing 7A; Scarf1: Scavenger receptor class F member 1; TMEM119: Transmembrane protein 119; OLFML3: Olfactomedin-like 3; SCL2A5: Solute carrier family 2 member 5; SALL1: Spalt-like transcription factor 1; CRIP1: Cysteine-rich intestinal protein 1; S100A8: S100 calciumbinding protein A8; S100A9: S100 calcium-binding protein A9; ANXA1: Annexin A1; CD14: Cluster of differentiation 14

\section{Acknowledgements}

We thank the Massachusetts General Hospital, Department of Pathology Flow and Image Cytometry Research Core, specifically Christina Luo and Ravi Mylvaganam for their help and execution of the flow cytometry experiments. Thorsten Mempel and Esteban Carrizosa are thanked for their suggestions regarding the flow cytometry setup used. We thank Sophie Dusoswa and Eric Tai for their help during some of the experiments. All members of the Breakefield, Tannous, Maguire, Bragg, and El Khoury labs that suggested ideas during lab meetings are very much appreciated.

\section{Authors' contributions}

J.E.K. conceived the study; S.L.N.M., X.O.B., and J.E.K. designed the experiments. S.L.N.M. and E.R.A. performed and analyzed experiments. X.Z. and S.P. assisted during animal experiments. S.L.N.M. prepared figures. C.P.L. and J.G. provided technical assistance. S.E.H. and P.S. performed RNAseq experiments and provided advice on microglial isolation. L.V.D.H. and L.M. performed immunofluorescent experiments and analyses. P.S., S.H., D.T., and S.S. assisted and performed RNA-seq experiments. S.L.N.M. and S.S. performed the computational and statistical analysis of RNA-seq data. X.O.B., M.L.D.B., and J.E.K. supervised the project. S.L.N.M, E.R.A., X.O.B., M.L.D.B., and J.E.K. wrote the manuscript. All authors edited or commented on the manuscript. The author(s) read and approved the final manuscript.

\section{Funding}

Sybren Maas acknowledges support from the Dutch Nijbakker-Morra travel stipend and the Dutch Cancer Society (KWF) travel grant. Xandra Breakefield acknowledges National Institutes of Health (NCI CA179563, CA069246 and CA232103) for funding used to perform this research. U19 CA179563 is supported by the National Institutes of Health Common Fund, through the Office of Strategic Coordination/Office of the NIH Director. Joseph El Khoury is funded by the National Institutes of Health (1RF1 AG051506, R01 Al119065). Generation of vectors used in this study was supported by the National Institutes of Health (NS045776) grant. The MGH Department of Pathology Flow and Image Cytometry Research Core obtained support from the National 
Institutes of Health Shared Instrumentation program (1S100D012027-01A1, 1S100D016372-01, 1S10RR020936-01, and 1S10RR023440-01A1).

\section{Availability of data and materials \\ Data availability}

Raw and processed transcriptomic data described in this manuscript are deposited in NCBI's Gene Expression Omnibus (GEO) and are accessible using GEO Series accession number GSE106775 at https://www.ncbi.nlm.nih. gov/geo/query/acc.cgi?acc $=$ GSE106775. Token for early data access: wdidoocgxxqjxsn

Code availability

$R$ scripts written for data processing and the generation of figures included in this manuscript are available online in a git repository. This includes the $R$ sessionInfo() data for compatibility information. The files and information can be accessed at https://github.com/sInmaas/Glioblastoma-Microglia-Project

\section{Ethics approval and consent to participate}

Animal experimentation was approved by the Massachusetts General Hospital Institution Animal Care and Use Committee and performed under IACUC protocol 2009 N000054.

\section{Consent for publication}

Not applicable.

\section{Competing interests}

The authors declare that they have no competing interests.

\section{Author details}

'Departments of Neurology and Radiology, Massachusetts General Hospital, Harvard Medical School, Boston, MA 02129, USA. ${ }^{2}$ Department of Neurosurgery, UMC Utrecht Brain Center, University Medical Center, Utrecht University, 3584 CX Utrecht, The Netherlands. ${ }^{3}$ Cancer Center, Massachusetts General Hospital, Harvard Medical School, Boston, MA 02129, USA. ${ }^{4}$ Center for Immunology \& Inflammatory Diseases, Massachusetts General Hospital, Harvard Medical School, Boston, MA 02129, USA. ${ }^{5}$ Center for Neuroscience and Cell Biology, University of Coimbra, 3004-517 Coimbra, Portugal. ${ }^{6}$ Department of Medicine, Massachusetts General Hospital, Harvard Medical School, Boston, MA 02129, USA. Institute of Atomic and Molecular Sciences/ Academia Sinica, 10617 Taipei, Taiwan. ${ }^{8}$ Department of Neurosurgery, Leiden University Medical Center, 2300 RC Leiden, The Netherlands. ${ }^{9}$ Department of Neurosurgery, Haaglanden Medical Center, 2512 VA The Hague, The Netherlands.

Received: 10 December 2019 Accepted: 31 March 2020 Published online: 16 April 2020

\section{References}

1. Stupp R, Hegi ME, Mason WP, van den Bent MJ, Taphoorn MJB, Janzer RC, Ludwin SK, Allgeier A, Fisher B, Belanger K, et al. Effects of radiotherapy with concomitant and adjuvant temozolomide versus radiotherapy alone on survival in glioblastoma in a randomised phase III study: 5-year analysis of the EORTC-NCIC trial. Lancet Oncol. 2009;10(5):459-66.

2. Reardon DA, Freeman G, Wu C, Chiocca EA, Wucherpfennig KW, Wen PY, Fritsch EF, Curry WT, Sampson JH, Dranoff G. Immunotherapy advances for glioblastoma. Neuro Oncol. 2014;16(11):1441-58.

3. Hambardzumyan D, Gutmann $\mathrm{DH}$, Kettenmann $\mathrm{H}$. The role of microglia and macrophages in glioma maintenance and progression. Nat Neurosci. 2016; 19(1):20-7.

4. Li W, Graeber MB. The molecular profile of microglia under the influence of glioma. Neuro Oncol. 2012;14(8):958-78

5. Morantz RA, Wood GW, Foster M, Clark M, Gollahon K. Macrophages in experimental and human brain tumors. Part 2: studies of the macrophage content of human brain tumors. J Neurosurg. 1979:50(3):305-11.

6. Morantz RA, Wood GW, Foster M, Clark M, Gollahon K. Macrophages in experimental and human brain tumors. Part 1: Studies of the macrophage content of experimental rat brain tumors of varying immunogenicity. J Neurosurg. 1979:50(3):298-304

7. Broekman ML, Maas SLN, Abels ER, Mempel TR, Krichevsky AM, Breakefield $\mathrm{XO}$. Multidimensional communication in the microenvirons of glioblastoma. Nat Rev Neurol. 2018;14(8):482-95.
8. Poon CC, Sarkar S, Yong VW, Kelly JJP. Glioblastoma-associated microglia and macrophages: targets for therapies to improve prognosis. Brain. 2017; 140(6):1548-60.

9. Markovic DS, Glass R, Synowitz M, Rooijen NV, Kettenmann H. Microglia stimulate the invasiveness of glioma cells by increasing the activity of metalloprotease-2. J Neuropathol Exp Neurol. 2005;64(9):754-62.

10. Markovic DS, Vinnakota K, Chirasani S, Synowitz M, Raguet H, Stock K, Sliwa M, Lehmann S, Kälin R, van Rooijen N, et al. Gliomas induce and exploit microglial MT1-MMP expression for tumor expansion. Proc Natl Acad Sci $U$ S A. 2009;106(30):12530-5.

11. Abels ER, Broekman ML, Breakefield XO, Maas SL. Glioma EVs contribute to immune privilege in the brain. Trends Cancer. 2019.

12. D'Asti E, Chennakrishnaiah S, Lee TH, Rak J. Extracellular vesicles in brain tumor progression. Cell Mol Neurobiol. 2016;36(3):383-407.

13. Maas SLN, Breakefield XO, Weaver AM. Extracellular vesicles: unique intercellular delivery vehicles. Trends Cell Biol. 2017;27(3):172-88.

14. Cocucci E, Meldolesi J. Ectosomes and exosomes: shedding the confusion between extracellular vesicles. Trends Cell Biol. 2015;25(6):364-72.

15. Abels ER, Breakefield XO. Introduction to extracellular vesicles: biogenesis, RNA cargo selection, content, release, and uptake. Cell Mol Neurobiol. 2016; 36(3):301-12.

16. Tkach $\mathrm{M}$, Théry $\mathrm{C}$. Communication by extracellular vesicles: where we are and where we need to go. Cell. 2016;164(6):1226-32

17. de Vrij J, Maas SL, Kwappenberg KM, Schnoor R, Kleijn A, Dekker L, Luider TM, de Witte LD, Litjens M, van Strien ME, et al. Glioblastoma-derived extracellular vesicles modify the phenotype of monocytic cells. Int J Cancer. 2015;137(7):1630-42

18. van der Vos KE, Abels ER, Zhang X, Lai C, Carrizosa E, Oakley D, Prabhakar S, Mardini O, Crommentuijn MHW, Skog J, et al. Directly visualized glioblastoma-derived extracellular vesicles transfer RNA to microglia/ macrophages in the brain. Neuro Oncol. 2016;18(1):58-69.

19. Abels ER, Maas SLN, Nieland L, Wei Z, Cheah PS, Tai E, Kolsteeg C-J, Dusoswa SA, Ting DT, Hickman S, et al. Glioblastoma-associated microglia reprogramming is mediated by functional transfer of extracellular miR-21. Cell Rep. 2019;28(12):3105-3119.e3107.

20. Lai CP, Kim EY, Badr CE, Weissleder R, Mempel TR, Tannous BA, Breakefield $X O$. Visualization and tracking of tumour extracellular vesicle delivery and RNA translation using multiplexed reporters. Nat Commun. 2015;6:7029.

21. Saederup N, Cardona AE, Croft K, Mizutani M, Cotleur AC, Tsou C-L, Ransohoff RM, Charo IF. Selective chemokine receptor usage by central nervous system myeloid cells in CCR2-red fluorescent protein knock-in mice. PloS One. 2010;5(10):e13693.

22. Sena-Esteves M, Tebbets JC, Steffens S, Crombleholme T, Flake AW. Optimized large-scale production of high titer lentivirus vector pseudotypes. I Virol Methods. 2004;122(2):131-9.

23. Lai CP, Mardini O, Ericsson M, Prabhakar S, Maguire CA, Chen JW, Tannous BA, Breakefield XO. Dynamic biodistribution of extracellular vesicles in vivo using a multimodal imaging reporter. ACS Nano. 2014;8(1):483-94.

24. McCabe JB, Berthiaume LG. Functional roles for fatty acylated aminoterminal domains in subcellular localization. Mol Biol Cell. 1999;10(11): $3771-86$.

25. Maas SLN, de Vrij J, van der Vlist EJ, Geragousian B, van Bloois L, Mastrobattista E, Schiffelers RM, Wauben MHM, Broekman MLD, Nolte-'t Hoen ENM. Possibilities and limitations of current technologies for quantification of biological extracellular vesicles and synthetic mimics. Control Release. 2015;200:87-96.

26. Hickman SE, Kingery ND, Ohsumi TK, Borowsky ML, Wang L-C, Means TK, El Khoury J. The microglial sensome revealed by direct RNA sequencing. Nat Neurosci. 2013:16(12):1896-905.

27. Love MI, Huber W, Anders S. Moderated estimation of fold change and dispersion for RNA-seq data with DESeq2. Genome Biol. 2014;15(12):550.

28. Liberzon A, Birger C, Thorvaldsdóttir H, Ghandi M, Mesirov JP, Tamayo P. The Molecular Signatures Database (MSigDB) hallmark gene set collection. Cell Syst. 2015;1(6):417-25.

29. Xue J, Schmidt SV, Sander J, Draffehn A, Krebs W, Quester I, De Nardo D, Gohel TD, Emde M, Schmidleithner L, et al. Transcriptome-based network analysis reveals a spectrum model of human macrophage activation. Immunity. 2014;40(2):274-88.

30. Chen H, Boutros PC. Venndiagram: a package for the generation of highlycustomizable Venn and Euler diagrams in R. BMC Bioinformatics. 2011;12(1):35 
31. Szulzewsky F, Arora S, de Witte L, Ulas T, Markovic D, Schultze JL, Holland EC, Synowitz M, Wolf SA, Kettenmann H. Human glioblastoma-associated microglia/monocytes express a distinct RNA profile compared to human control and murine samples. Glia. 2016;64(8):1416-36.

32. Darmanis S, Sloan SA, Croote D, Mignardi M, Chernikova S, Samghababi P, Zhang Y, Neff N, Kowarsky M, Caneda C, et al. Single-cell RNA-Seq analysis of infiltrating neoplastic cells at the migrating front of human glioblastoma. Cell Rep. 2017;21(5):1399-410.

33. Hickman SE, Allison EK, El Khoury J. Microglial dysfunction and defective beta-amyloid clearance pathways in aging Alzheimer's disease mice. J Neurosci. 2008;28(33):8354-60.

34. Hickman SE, El Khoury J. Analysis of the microglial sensome. Methods Mol Biol. 2019;2034:305-23.

35. Ginhoux F, Jung S. Monocytes and macrophages: developmental pathways and tissue homeostasis. Nat Rev Immunol. 2014;14(6):392-404.

36. Greter M, Lelios I, Croxford AL. Microglia versus myeloid cell nomenclature during brain inflammation. Front Immunol. 2015;6(7540):249.

37. Ransohoff RM. A polarizing question: do $M 1$ and $M 2$ microglia exist? Nat Neurosci. 2016;19(8):987-91.

38. Hickman S, Izzy S, Sen P, Morsett L, El Khoury J. Microglia in neurodegeneration. Nat Neurosci. 2018;21(10):1359-69.

39. Jassam YN, Izzy S, Whalen M, McGavern DB, El Khoury J. Neuroimmunology of traumatic brain injury: time for a paradigm shift. Neuron. 2017;95(6):1246-65.

40. Ransohoff RM, El Khoury J. Microglia in health and disease. Cold Spring Harb Perspect Biol. 2015;8(1):a020560.

41. Mariani MM, Kielian T. Microglia in infectious diseases of the central nervous system. J Neuroimmune Pharmacol. 2009;4(4):448-61.

42. Salter MW, Stevens B. Microglia emerge as central players in brain disease. Nat Med. 2017;23(9):1018-27.

43. Bialas AR, Stevens B. TGF- $\beta$ signaling regulates neuronal C1q expression and developmental synaptic refinement. Nat Neurosci. 2013;16(12):1773-82.

44. Butovsky O, Jedrychowski MP, Moore CS, Cialic R, Lanser AJ, Gabriely G, Koeglsperger T, Dake B, Wu PM, Doykan CE, et al. Identification of a unique TGF- $\beta$-dependent molecular and functional signature in microglia. Nat Neurosci. 2014;17(1):131-43.

45. Krasemann S, Madore C, Cialic R, Baufeld C, Calcagno N, El Fatimy R, Beckers L, O'Loughlin E, Xu Y, Fanek Z, et al. The TREM2-APOE pathway drives the transcriptional phenotype of dysfunctional microglia in neurodegenerative diseases. Immunity. 2017:47(3):566-581.e569

46. Chao MP, Jaiswal S, Weissman-Tsukamoto R, Alizadeh AA, Gentles AJ, Volkmer J, Weiskopf K, Willingham SB, Raveh T, Park CY, et al. Calreticulin is the dominant pro-phagocytic signal on multiple human cancers and is counterbalanced by CD47. Sci Translat Med. 2010;2(63):63ra94.

47. Fife BT, Pauken KE, Eagar TN, Obu T, Wu J, Tang Q, Azuma M, Krummel MF, Bluestone JA. Interactions between PD-1 and PD-L1 promote tolerance by blocking the TCR-induced stop signal. Nat Immunol. 2009;10(11):1185-92.

48. Heiland DH, Haaker G, Delev D, Mercas B, Masalha W, Heynckes S, Gäbelein A, Pfeifer D, Carro MS, Weyerbrock A, et al. Comprehensive analysis of PD-L1 expression in glioblastoma multiforme. Oncotarget. 2017;8(26):42214-25

49. Schachtele SJ, Hu S, Sheng WS, Mutnal MB, Lokensgard JR. Glial cells suppress postencephalitic CD8+ T lymphocytes through PD-L1. Glia. 2014; 62(10):1582-94.

50. Bardhan K, Anagnostou T, Boussiotis VA. The PD1:PD-L1/2 pathway from discovery to clinical implementation. Front Immunol. 2016;7(1):550.

51. Brahmer JR, Tykodi SS, Chow LQM, Hwu W-J, Topalian SL, Hwu P, Drake CG, Camacho LH, Kauh J, Odunsi K, et al. Safety and activity of anti-PD-L1 antibody in patients with advanced cancer. N Engl J Med. 2012;366(26):2455-65.

52. Davalos D, Grutzendler J, Yang G, Kim JV, Zuo Y, Jung S, Littman DR, Dustin ML, Gan W-B. ATP mediates rapid microglial response to local brain injury in vivo. Nat Neurosci. 2005;8(6):752-8.

53. Nimmerjahn A, Kirchhoff F, Helmchen F. Resting microglial cells are highly dynamic surveillants of brain parenchyma in vivo. Science. 2005;308(5726): 1314-8.

54. Kopatz J, Beutner C, Welle K, Bodea LG, Reinhardt J, Claude J, LinnartzGerlach B, Neumann H. Siglec-h on activated microglia for recognition and engulfment of glioma cells. Glia. 2013;61(7):1122-33.

55. Kitamura H, Makide K, Shuto A, Ikubo M, Inoue A, Suzuki K, Sato $Y$, Nakamura S, Otani Y, Ohwada T, et al. GPR34 is a receptor for lysophosphatidylserine with a fatty acid at the sn-2 position. J Biochem. 2012;151(5):511-8.
56. Riedl S, Rinner B, Asslaber M, Schaider H, Walzer S, Novak A, Lohner K, Zweytick D. In search of a novel target - phosphatidylserine exposed by non-apoptotic tumor cells and metastases of malignancies with poor treatment efficacy. Biochim Biophys Acta. 2011;1808(11):2638-45.

57. Eibinger G, Fauler G, Bernhart E, Frank S, Hammer A, Wintersperger A, Eder $H$, Heinemann A, Mischel PS, Malle E, et al. On the role of 25hydroxycholesterol synthesis by glioblastoma cell lines. Implications for chemotactic monocyte recruitment. Exp Cell Res. 2013;319(12):1828-38.

58. van der Putten C, Zuiderwijk-Sick EA, van Straalen L, de Geus ED, Boven LA, Kondova I, ljzerman AP, Bajramovic JJ. Differential expression of adenosine A3 receptors controls adenosine A2A receptor-mediated inhibition of TLR responses in microglia. J Immunol. 2009;182(12):7603-12.

59. Cheng W, Ren X, Zhang C, Cai J, Liu Y, Han S, Wu A. Bioinformatic profiling identifies an immune-related risk signature for glioblastoma. Neurology. 2016:86(24):2226-34

60. Feng X, Szulzewsky F, Yerevanian A, Chen Z, Heinzmann D, Rasmussen RD, Alvarez-Garcia V, Kim Y, Wang B, Tamagno I, et al. Loss of CX3CR1 increases accumulation of inflammatory monocytes and promotes gliomagenesis. Oncotarget. 2015:6(17):15077-94.

61. Morrone FB, Oliveira DL, Gamermann P, Stella J, Wofchuk S, Wink MR, Meurer L, Edelweiss MIA, Lenz G, Battastini AMO. In vivo glioblastoma growth is reduced by apyrase activity in a rat glioma model. BMC Cancer. 2006;6(1):226

62. Tozaki-Saitoh H, Miyata H, Yamashita T, Matsushita K, Tsuda M, Inoue K. P2Y12 receptors in primary microglia activate nuclear factor of activated Tcell signaling to induce C-C chemokine 3 expression. J Neurochem. 2017; 141(1):100-10.

63. Lin H-C, Song T-Y, Hu M-L. S-Adenosylhomocysteine promotes the invasion of C6 glioma cells via increased secretion of matrix metalloproteinase-2 in murine microglial BV2 cells. Toxicol Sci. 2009;112(2):322-30.

64. PrabhuDas MR, Baldwin CL, Bollyky PL, Bowdish DME, Drickamer K, Febbraio M, Herz J, Kobzik L, Krieger M, Loike J, et al. A consensus definitive classification of scavenger receptors and their roles in health and disease. J Immunol. 2017:3775-89.

65. Mirzaei R, Sarkar S, Yong WW. T Cell exhaustion in glioblastoma: intricacies of immune checkpoints. Trends Immunol. 2017;38(2):104-15.

66. Ren SX, Cheng ASL, To KF, Tong JHM, Li MS, Shen J, Shen J, Wong CCM, Zhang L, Chan RLY, et al. Host immune defense peptide LL-37 activates caspase-independent apoptosis and suppresses colon cancer. Cancer Res. 2012;72(24):6512-23.

67. Chen X, Qi G, Qin M, Zou Y, Zhong K, Tang Y, Guo Y, Jiang X, Liang L, Zou $X$. DNA methylation directly downregulates human cathelicidin antimicrobial peptide gene (CAMP) promoter activity. Oncotarget. 2017; 8(17):27943-52.

68. Raza SM, Lang FF, Aggarwal BB, Fuller GN, Wildrick DM, Sawaya R. Necrosis and glioblastoma: a friend or a foe? A review and a hypothesis. Neurosurgery. 2002:51(1):2-12 discussion 12-13.

69. Okawa S, Gagrica S, Blin C, Ender C, Pollard SM, Krijgsveld J. Proteome and secretome characterization of glioblastoma-derived neural stem cells. Stem Cells. 2017:35(4):967-80.

70. Sin WC, Aftab Q, Bechberger JF, Leung JH, Chen H, Naus CC. Astrocytes promote glioma invasion via the gap junction protein connexin43. Oncogene. 2016;35(12):1504-16.

71. Verhaak RG, Hoadley KA, Purdom E, Wang V, Qi Y, Wilkerson MD, Miller CR, Ding L, Golub T, Mesirov JP, et al. Integrated genomic analysis identifies clinically relevant subtypes of glioblastoma characterized by abnormalities in PDGFRA, IDH1, EGFR, and NF1. Cancer Cell. 2010;17(1):98-110.

\section{Publisher's Note}

Springer Nature remains neutral with regard to jurisdictional claims in published maps and institutional affiliations. 